\title{
Quadratic forms classify products on quotient ring spectra
}

\author{
ALAIN JEANNERET \\ SAMUEL WÜTHRICH
}

\begin{abstract}
We construct a free and transitive action of the group of bilinear forms $\operatorname{Bil}\left(I / I^{2}[1]\right)$ on the set of $R$-products on $F$, a regular quotient of an even $E_{\infty}$-ring spectrum $R$ with $F_{*} \cong R_{*} / I$. We show that this action induces a free and transitive action of the group of quadratic forms $\mathrm{QF}\left(I / I^{2}[1]\right)$ on the set of equivalence classes of $R$-products on $F$. The characteristic bilinear form of $F$ introduced by the authors in a previous paper is the natural obstruction to commutativity of $F$. We discuss the examples of the Morava $K$-theories $K(n)$ and the 2-periodic Morava $K$-theories $K_{n}$.
\end{abstract}

55P42, 55P43, 55U20; 18E30

\section{Introduction}

With the advent of sound foundations for a theory of modules over an $E_{\infty}$-ring spectrum $R$ (for instance as developed by Elmendorf, Kriz, Mandell and May [4]), it has become possible to mimic in homotopy theory well-known constructions usually performed in algebra. The setting is the homotopy category $\mathscr{D}_{R}$ of $R$-module spectra over $R$, a category equipped with a smash product $\wedge_{R}$ (the equivalent of the tensor product), giving $\mathscr{D}_{R}$ the structure of a symmetric monoidal category. Objects in $\mathscr{D}_{R}$ may be regarded as ordinary spectra by neglect of structure, via a (lax) monoidal functor to the classical stable homotopy category.

With this framework at hand, the problem of constructing quotient spectra, ie spectra whose homotopy groups are isomorphic to a given quotient of the coefficient ring $R_{*}=\pi_{*}(R)$ of $R$, admits a clean and transparent solution for a large class of quotients. The quotients in question are the quotients $R_{*} / I$ by ideals $I$ which are generated by regular sequences. The $R$-module spectra realizing such quotients are often referred to as regular quotients.

Shortly after the publication of [4], Strickland [9] proved that for $E_{\infty}$-ring spectra $R$ for which $R_{*}$ forms a domain and which is trivial in odd degrees, any regular quotient can be realized as an $R$-ring spectrum, ie as a monoid in $\mathscr{D}_{R}$, and therefore in particular as a ring spectrum. 
The aim of the present article is to give a conceptual description of the set of all $R$-ring structures on regular quotients $F$ of $R$, as well as of the set of equivalence classes of $R$-ring structures. Our result in both cases is based on a free and transitive action of a certain abelian group canonically associated to $F$ on the set of products.

As an application, we show that the characteristic bilinear form $b_{F}$ of a regular quotient $F$, introduced by the authors in [5], is always symmetric and provides a measure for the noncommutativity of $F$.

As another application, we give a necessary and sufficient criterion for a map of regular quotient rings $\pi: F \rightarrow G$ to be multiplicative, in terms of the characteristic bilinear forms.

We use our results to classify products on the 2-periodic Morava $K$-theories $K_{n}$, which from an algebro-geometric point of view are the more natural objects to study than their classical variants $K(n)$. In contrast to $K(n)$, we show that $K_{n}$ supports a large number of products, even many commutative ones for $p$ odd and $n>1$.

In addition, we confirm many well-known facts concerning certain families of quotients of complex cobordism $M U$, whose existing proofs are in many cases technically forbidding and scattered in the literature.

We now proceed to a more detailed overview of the content of this article. Throughout, $R$ denotes an $E_{\infty}$-ring spectrum for which $R_{*}$ is a domain and is trivial in odd degrees.

The following result assembles our two main theorems (Theorem 4.1 and Theorem 7.2). The symbol $I / I^{2}[1]$ stands for the graded module $I / I^{2}$ shifted by one, where $I \subseteq R_{*}$ is an ideal.

Theorem 1 Let $F$ be a regular quotient of $R$ with coefficients $F_{*} \cong R_{*} / I$.

(i) The abelian group $\operatorname{Bil}\left(I / I^{2}[1]\right)$ of bilinear forms on $I / I^{2}[1]$ acts in a natural way freely and transitively on the set of $R$-products on $F$.

(ii) This action induces a free and transitive action of the abelian group $\mathrm{QF}\left(I / I^{2}[1]\right)$ of quadratic forms on $I / I^{2}[1]$ on the set of equivalence classes of $R$-products on $F$.

For a regular quotient ring $F=R / I$ with product $\mu$ and a bilinear form $\beta \in$ $\operatorname{Bil}\left(I / I^{2}[1]\right)$, we will denote by $\beta F$ the $R$-module $F$, endowed with the product $\beta \mu$ in the sequel.

For the proof of the theorem we build on our previous paper [5]. The central ingredient is the module of (homotopy) derivations $\operatorname{Der}_{R}^{*}(F)$. Of crucial importance is the fact 
proved in [5] that $\operatorname{Der}_{R}^{*}(F)$ does not depend on the product of $F$, as a submodule of the algebra of endomorphisms $F_{R}^{*}(F)$.

Applied to $R=\widehat{E}(n)$, the completed Johnson-Wilson theories, and $F=K(n)$, the theorem implies immediately that for $p$ odd, there is precisely one $\widehat{E}(n)$-product on $K(n)$, which therefore must be commutative. For $p=2$, it implies that there are precisely two nonequivalent $\widehat{E}(n)$-products on $K(n)$. They are both noncommutative, as we will see below. These are well-known results.

For $R=E_{n}$, the Morava $E$-theories, and $F=K_{n}$, we deduce that there are $p^{n} n^{2}$ different $E_{n}$-products and $p^{n} \frac{n}{2}(n+1)$ equivalence classes of $E_{n}$-products on $K_{n}$.

It is natural to ask whether there is an invariant which distinguishes the different products on $F$ or at least the different equivalence classes of products. A candidate is the characteristic bilinear form

$$
b_{F}: I / I^{2}[1] \otimes_{F_{*}} I / I^{2}[1] \longrightarrow F_{*}
$$

of a regular quotient ring $F$ constructed in [5]. We prove as Corollary 7.5:

Proposition 2 The characteristic bilinear forms of equivalent products on $F$ coincide. The converse holds whenever $F_{*}$ is 2-torsion free.

In fact, the characteristic bilinear form $b_{F}$ admits a natural characterization in terms of the action of the theorem. To express it, let $F^{\text {op }}$ denote the opposite ring of $F$. We prove as Corollary 5.5:

Proposition 3 The characteristic bilinear form $b_{F}$ of a regular quotient ring $F$ satisfies $F^{\mathrm{op}}=b_{F} F$.

Hence $b_{F}$ is the obstruction to commutativity of $F$ :

Corollary 4 A regular quotient ring $F$ is commutative if and only if $b_{F}=0$.

Consider again the Morava $K$-theories $K(n)$ at $p=2$. We proved in [5] that it admits an $\hat{E}(n)$-product $\mu$ with nontrivial characteristic bilinear form. Corollary 4 implies that $\mu$ cannot be commutative. Therefore the second product on $K(n)$ is neither, as it must be the opposite of $\mu$. Moreover, Proposition 3 recovers the formula from Nassau [6] (see Section 8 for the definition of $v_{n}$ and $Q_{n-1}$ )

$$
\mu^{\mathrm{op}}=\mu \circ\left(1+v_{n} Q_{n-1} \wedge Q_{n-1}\right) .
$$


As a consequence of Theorem 1, there is in general a large variety of products on $F$, even up to equivalence, unless there are only few bilinear forms on $I / I^{2}[1]$ due to sparseness of the coefficients $F_{*}$. One may ask if the situation changes when one restricts to commutative products. To approach this question, one needs a formula which expresses how $b_{F}$ transforms under the action of $\operatorname{Bil}\left(I / I^{2}[1]\right)$, in view of Corollary 4. Let $\beta^{t}$ denote the transpose of a bilinear form $\beta$ on $I / I^{2}[1]$, defined by $\beta^{t}(x \otimes y)=\beta(y \otimes x)$. We prove as Corollary 5.3:

Proposition 5 Let $F$ be a regular quotient ring with characteristic bilinear form $b_{F}$ and let $\beta$ be a bilinear form in $\operatorname{Bil}\left(I / I^{2}[1]\right)$. Then the characteristic bilinear form of $\beta F$ is given by $b_{\beta F}=b_{F}-\beta-\beta^{t}$.

With Corollary 4, it follows that for commutative $F, \beta F$ is commutative if and only if $\beta$ is antisymmetric. Together with Theorem 1 , this implies the following result (Proposition 7.8, Corollary 7.10), which sharpens a result of [9].

Corollary 6 Let $F$ be a regular quotient ring of $R$. If $2 \in F_{*}$ is invertible, there exists a unique commutative product on $F$ up to equivalence. If $F_{*}$ is 2-torsion free, there exists at most one commutative product on $F$ up to equivalence.

For the 2-periodic Morava $K$-theories, Proposition 5 implies that there are $p^{n} \frac{n}{2}(n-1)$ commutative $E_{n}$-products for odd $p$, all of which are equivalent. At the prime $2, K_{n}$ admits a product with nontrivial characteristic bilinear form, by a result from [5]. From this, it follows that there does not exists any commutative product on $K_{n}$ for $p=2$.

Using the fact proved in [9] that the Brown-Peterson spectrum $B P$ at a prime $p$ admits a commutative $M U$-product, it follows that there is a unique commutative $M U$-product on $B P$ up to equivalence.

The products on regular quotients $F$ constructed in [9] have a very special form. To explain in what sense, let $\left(x_{1}, x_{2}, \ldots\right)$ be a regular sequence generating $I$, where $F_{*} \cong R_{*} / I$. Then $F$ is equivalent to $R / x_{1} \wedge_{R} R / x_{2} \wedge_{R} \cdots$ as an $R$-module spectrum (see Section 2 for details). The products considered in [9] are all obtained by "smashing together" products on the $R$-module spectra $R / x_{k}$. We call such products diagonal and refer to products equivalent to diagonal ones as diagonalizable. In [5], we showed that the characteristic bilinear form of a diagonal regular quotient ring is diagonal. Together with Proposition 5, this implies (Corollary 5.4):

Corollary 7 The characteristic bilinear form $b_{F}$ of a regular quotient ring $F$ is symmetric. 
The following result is proved as Proposition 7.13:

Proposition 8 Assume that $R_{*}$ is a finite-dimensional regular local ring with maximal ideal $I$ and suppose that $F$ is an $R$-ring satisfying $F_{*} \cong R_{*} / I$. If the characteristic $p$ of $F_{*}$ is zero or an odd prime, then $F$ is diagonalizable. If $p=2$, then $F$ is diagonalizable unless $b_{F}$ is alternating and nontrivial, in which case $F$ is not diagonalizable.

This implies for instance that any $E_{n}$-product on $K_{n}$ is diagonalizable, for $p$ arbitrary. However, not every regular quotient ring is diagonalizable: We construct a nondiagonalizable $M U$-ring spectrum in Section 8.

As an application of Theorem 1, we give a necessary and sufficient condition for a map $\pi: F \rightarrow G$ between regular quotients of $R$ to be multiplicative. Let $I \subseteq J$ be the ideals of $R_{*}$ for which $F_{*} \cong R_{*} / I$ and $G_{*}=R_{*} / J$, respectively. In [5], we introduced a bilinear form

$$
b_{F}^{G}:\left(G_{*} \otimes_{F_{*}} I / I^{2}[1]\right) \otimes_{G_{*}}\left(G_{*} \otimes_{F_{*}} I / I^{2}[1]\right) \rightarrow G_{*},
$$

which depends on $\pi$. Let $b_{F}$ and $b_{G}$ denote the characteristic bilinear forms of $F$ and $G$, respectively. Let $\pi^{*}\left(b_{G}\right)$ be the bilinear form on $G_{*} \otimes_{F_{*}} I / I^{2}[1]$ obtained by "pulling back" $b_{G}$ along the morphism $\bar{\pi}: I / I^{2}[1] \rightarrow J / J^{2}[1]$ induced by $\pi$.

Theorem 9 Suppose that $\pi: F \rightarrow G$ is as above and assume that the induced map $G_{*} \otimes_{F_{*}} I / I^{2}[1] \rightarrow J / J^{2}[1]$ is split injective. Then $\pi$ is multiplicative if and only if $G_{*} \otimes b_{F}=b_{F}^{G}=\pi^{*}\left(b_{G}\right)$.

As an illustration, we show that there are infinitely many $M U$-products on the spectrum $P(n)$ for any prime $p$ such that the canonical map $B P \rightarrow P(n)$ is multiplicative, where $B P$ is endowed with an arbitrary commutative $M U$-product (see Section 8).

Acknowledgements The second author would like to thank Professor Kathryn Hess for her support throughout his time at the EPFL in Lausanne.

\section{Notation and conventions}

In this article, we will work in the framework of $\mathbb{S}$-modules of [4]. In this setting, $E_{\infty}$-ring spectra correspond to commutative $\mathbb{S}$-algebras. Throughout, $R$ denotes an even commutative $\mathbb{S}$-algebra, ie one with $R_{\text {odd }}=0$. We also assume that the coefficient ring $R_{*}$ of $R$ is a domain (see [5, Remark 2.11]). Associated to $R$ is the homotopy category $\mathscr{D}_{R}$ of $R$-module spectra. For simplicity, we refer to its objects as 
$R$-modules. The smash product $\wedge_{R}$ endows $\mathscr{D}_{R}$ with a symmetric monoidal structure. We will abbreviate $\wedge_{R}$ by $\wedge$ throughout the paper.

Monoids in $\mathscr{D}_{R}$ are called $R$-ring spectra or just $R$-rings. Unless otherwise specified, we use the generic notation $\eta_{F}: R \rightarrow F$ (or simply $\eta$ ) for the unit and $\mu_{F}: F \wedge F \rightarrow F$ (or simply $\mu$ ) for the multiplication of an $R$-ring $F$. Mostly, $\eta_{F}$ will be clear from the context, in which case we call a map $\mu_{F}: F \wedge F \rightarrow F$ which gives $F$ the structure of an $R$-ring an $R$-product or just a product. For a given $R$-ring $\left(F, \mu_{F}, \eta_{F}\right)$, we will often be in the situation where we consider another product $\bar{\mu}_{F}$ on $F$. We then write $\bar{F}$ for the $R$-ring $\left(F, \bar{\mu}_{F}, \eta_{F}\right)$. We denote the opposite of an $R$-ring $F$ by $F^{\text {op }}$. Its product is given by $\mu_{F^{\text {op }}}=\mu_{F} \circ \tau$, where $\tau: F \wedge F \rightarrow F \wedge F$ is the switch map.

An $R$-ring $\left(F, \mu_{F}, \eta_{F}\right)$ determines multiplicative homology and cohomology theories $F_{*}^{R}(-)=\pi_{*}(F \wedge-)=\mathscr{D}_{R}^{-*}(R, F \wedge-)$ and $F_{R}^{*}(-)=\mathscr{D}_{R}^{*}(-, F)$, respectively, on $\mathscr{D}_{R}$. For an $R$-module $M$, the homology $F_{*}^{R}(M)$ is an $F_{*}$-bimodule in a natural way. Even if $F_{*}$ is commutative, the left and right $F_{*}$-actions may well be different. However, if we assume that $F$ is a quotient of $R$, by which we mean that the unit map $\eta_{F}$ induces a surjection on homotopy groups (see Section 2 below for definitions), the left and right $F_{*}$-actions agree. In this case, we can refer to $F_{*}^{R}(M)$ as a $F_{*}-$ module without any ambiguity. A similar discussion applies to cohomology $F_{R}^{*}(M)$. See Section 1.1 of [5] for a more detailed discussion.

Denote by $M_{*}[d]$ the $d$-fold suspension of a graded abelian group $M_{*}$, so $\left(M_{*}[d]\right)_{k}=$ $M_{k-d}$. With this convention, we have $\left(\Sigma^{d} M\right)_{*}=M_{*}[d]$ for an $R$-module $M$. We use the convention $M^{*}=M_{-*}$. If the ground ring is clear from the context, we omit it from the tensor product symbol $\otimes$ from now on. We write $D_{F_{*}}\left(M_{*}\right)$ or just $D\left(M_{*}\right)$ for the dual $\operatorname{Hom}_{F_{*}}^{*}\left(M_{*}, F_{*}\right)$ of a graded module $M_{*}$ over a graded ring $F_{*}$.

We introduce some notation and recall some well-known facts concerning bilinear and quadratic forms. For an $F_{*}$-module $V$, we write $\operatorname{Bil}(V)$ for the abelian group of (degree zero) bilinear forms on $V$. For $\beta \in \operatorname{Bil}(V)$, we set $\beta^{t}(x \otimes y)=\beta(y \otimes x)$ for $x, y \in V$. A bilinear form $\beta \in \operatorname{Bil}(V)$ is symmetric if $\beta^{t}=\beta$, antisymmetric if $\beta^{t}=-\beta$ and alternating if $\beta(v \otimes v)=0$ for any $v \in V$. We write $\operatorname{Sym}(V)$, $\operatorname{Asym}(V)$ and $\operatorname{Alt}(V)$ for the subgroups of $\operatorname{Bil}(V)$ consisting of the symmetric, antisymmetric and alternating bilinear forms, respectively. If $V$ is 2-torsion-free, we have $\operatorname{Sym}(V) \cap \operatorname{Alt}(V)=0$ and $\operatorname{Asym}(V)=\operatorname{Alt}(V)$. If $2 \in F_{*}$ is invertible, we have the usual decomposition $\operatorname{Bil}(V)=\operatorname{Sym}(V) \oplus \operatorname{Alt}(V)$.

Let $\mathrm{QF}(V)$ denote the group of quadratic forms $q: V \rightarrow F_{*}$. Recall that the grading convention is that $|q(v)|=2 n$ for $v \in V_{n}$. For $\beta \in \operatorname{Bil}(V), q(v)=\beta(v \otimes v)$ is easily seen to be a quadratic form. We therefore obtain a group homomorphism $\chi: \operatorname{Bil}(V) \rightarrow \mathrm{QF}(V)$, whose kernel is $\operatorname{Alt}(V)$. If $V$ is $F_{*}-$ free, $\chi$ is surjective 
(see Bourbaki [3, Chapter IX, Section 3, Proposition 2]) and so we have a canonical isomorphism $\operatorname{Bil}(V) / \operatorname{Alt}(V) \cong \mathrm{QF}(V)$. If $2 \in F_{*}$ is invertible, we recover the wellknown isomorphism $\operatorname{Sym}(V) \cong \mathrm{QF}(V)$.

For a ring homomorphism $\pi_{*}: F_{*} \rightarrow k_{*}$ and $\beta \in \operatorname{Bil}(V)$, we define $k_{*} \otimes \beta$ to be the bilinear form on the $k_{*}$-module $k_{*} \otimes F_{*} V$ determined by

$$
\left(k_{*} \otimes \beta\right)((1 \otimes x) \otimes(1 \otimes y))=\pi_{*}(\beta(x \otimes y))
$$

for $x, y \in V$. If $\pi_{*}: W \rightarrow V$ is a morphism of $F_{*}$-modules and $\beta \in \operatorname{Bil}(V), \pi^{*}(\beta)$ denotes the bilinear form on $W$ which on $x, y \in W$ takes the value

$$
\pi^{*}(\beta)(x \otimes y)=\beta\left(\pi_{*}(x) \otimes \pi_{*}(y)\right) .
$$

\section{Recollection}

In this section we collect some results, definitions and notation from [5] which we are using in the present paper.

A quotient module of $R$ is an $R$-module $F$ with a map $\eta_{F}: R \rightarrow F$ of $R$-modules which induces a surjection on homotopy groups, that is $F_{*} \cong R_{*} / I$. We will write $F=R / I$ for such an $F$ in the sequel. The modules of interest for our purposes are the regular quotient modules of $R$. By this, we mean quotient modules $F=R / I$ whose ideal $I$ is generated by some (finite or infinite) regular sequence $\left(x_{1}, x_{2}, \ldots\right)$ in $R_{*}$.

A (regular) quotient ring of $R$ is an $R$-ring $\left(F, \mu_{F}, \eta_{F}\right)$ with product $\mu_{F}$ such that $\left(F, \eta_{F}\right)$ is a (regular) quotient module of $R$. For instance, let $F=R / I$ be a regular quotient of $R$ and $\left(x_{1}, x_{2}, \ldots\right)$ a regular sequence generating the ideal $I$. Then $F$ is isomorphic in $\mathscr{D}_{R}$ to

$$
R / x_{1} \wedge R / x_{2} \wedge \cdots:=\operatorname{hocolim}_{k} R / x_{1} \wedge \cdots \wedge R / x_{k},
$$

where for $x \in R_{*}$, we denote by $R / x$ the homotopy cofiber of $x: \Sigma^{|x|} R \rightarrow R$. For any products $\mu_{i}$ on $R / x_{i}$, there is a uniquely determined product $\mu$ on $F=R / I$ such that the natural maps $j_{i}: R / x_{i} \rightarrow F$ are multiplicative and commute for $k \neq l$, ie $\mu\left(j_{k} \wedge j_{l}\right)=\mu^{\mathrm{op}}\left(j_{k} \wedge j_{l}\right)$. This ring $F$ is called the smash ring spectrum of the $R / x_{i}$. If we need to be more precise, we refer to the product map $\mu_{F}$ as the smash ring product of the $\mu_{i}$. A regular quotient ring $F$ whose product is of this form is said to be diagonal or diagonal with respect to $\left(x_{1}, x_{2}, \ldots\right)$ if we need to keep track of the regular sequence.

An admissible pair is by definition a triple $(F, k, \pi)$ consisting of two quotient $R$-rings $\left(F, \mu_{F}, \eta_{F}\right),\left(k, \mu_{k}, \eta_{k}\right)$ and a unital $R$-module map $\pi: F \rightarrow k$, ie an 
$R$-morphism $\pi$ with $\pi \eta_{F}=\eta_{k}$. If $\pi$ is a map of $R$-ring spectra, we call $(F, k, \pi)$ a multiplicative admissible pair. A typical example of an admissible pair is $\left(F, F, 1_{F}\right)$ where $1_{F}$ is the identity on $F$, but where we distinguish two products $\mu$ and $\nu$ on $F$.

In the following, we fix an admissible pair Its characteristic homomorphism is a homomorphism of $F_{*}$-modules

$$
\varphi_{F}^{k}: I / I^{2}[1] \longrightarrow k_{*}^{R}(F),
$$

which is natural in $F$ and $k$ and independent of the products on $F$ and $k$.

The homology group $k_{*}^{R}(F)$ carries a natural $k_{*}$-algebra structure, whose product is defined by the following composition of $k_{*}$-homomorphisms

$$
m_{F}^{k}: k_{*}^{R}(F) \otimes_{k_{*}} k_{*}^{R}(F) \stackrel{\kappa_{k}}{\longrightarrow} k_{*}^{R}(F \wedge F) \stackrel{k_{*}^{R}\left(\mu_{F}\right)}{\longrightarrow} k_{*}^{R}(F) .
$$

Here $\kappa_{k}$ stands for the Künneth homomorphism associated to the ring $k$.

The characteristic bilinear form $b_{F}^{k}$ associated to $(F, k, \pi)$ is defined as the following composition of $k_{*}$-homomorphisms

$$
b_{F}^{k}:\left(k_{*} \otimes_{F_{*}} I / I^{2}[1]\right)^{\otimes 2} \stackrel{{ }^{\pi} \varphi^{\otimes 2}}{\longrightarrow} k_{*}^{R}(F)^{\otimes 2} \stackrel{m_{F}^{k}}{\longrightarrow} k_{*}^{R}(F) \stackrel{k_{*}(\pi)}{\longrightarrow} k_{*}^{R}(k) \stackrel{\left(\mu_{k}\right)_{*}}{\longrightarrow} k_{*},
$$

where ${ }^{\pi} \varphi$ is the $k_{*}$-homomorphism canonically induced by $\varphi$. The characteristic quadratic form $q_{F}^{k}$ is defined as $q_{F}^{k}(\bar{x})=b_{F}^{k}(\bar{x} \otimes \bar{x})$ for $\bar{x} \in k_{*} \otimes_{F_{*}} I / I^{2}[1]$.

We write $\varphi_{F}, b_{F}$ and $q_{F}$ for the characteristic homomorphism, bilinear and quadratic forms of the admissible pair $\left(F, F, 1_{F}\right)$, respectively, for a quotient ring $F$.

If $(F, k, \pi)$ is multiplicative, the characteristic bilinear form $b_{F \text { op }}^{k}$ of the associated admissible pair $\left(F^{\mathrm{op}}, k, \pi\right)$ is trivial. In particular, $b_{F \text { op }}^{F}=0$ for a quotient ring $F$ and $b_{F}=0$ for a commutative quotient ring $F$.

The characteristic homomorphism $\varphi=\varphi_{F}^{k}$ lifts to an algebra homomorphism

$$
\Phi: \mathcal{C} \ell\left(k_{*} \otimes_{F_{*}} I / I^{2}[1], q_{F}^{k}\right) \longrightarrow k_{*}^{R}(F),
$$

where $\mathcal{C} \ell\left(k_{*} \otimes_{F_{*}} I / I^{2}[1], q_{F}^{k}\right)$ denotes the Clifford algebra of the quadratic module $\left(k_{*} \otimes_{F_{*}} I / I^{2}[1], q_{F}^{k}\right)$. If $F$ is a regular quotient, then $\Phi$ is an isomorphism. In particular, this yields an algebra isomorphism

$$
F_{*}^{R}\left(F^{\mathrm{op}}\right) \cong \Lambda\left(I / I^{2}[1]\right) .
$$

To be more explicit, fix a regular sequence $\left(x_{1}, x_{2}, \ldots\right)$ generating $I$. This choice determines an isomorphism $I / I^{2}[1] \cong \bigoplus_{i} F_{*} \bar{x}_{i}$, where $\bar{x}_{i}$ denotes the residue class 
of $x_{i}$ in $I / I^{2}[1]$. Letting $a_{i}=\varphi_{F \text { op }}^{F}\left(\bar{x}_{i}\right) \in F_{*}^{R}(F)$, we have

$$
F_{*}^{R}\left(F^{\mathrm{op}}\right) \cong \Lambda\left(a_{1}, a_{2}, \ldots\right) .
$$

If $(F, k, \pi)$ is multiplicative, we may consider the module of (homotopy) derivations $\operatorname{Der}_{R}^{*}(F, k) \subseteq k_{R}^{*}(F)$. By definition, these are maps $d: F \rightarrow \Sigma^{i} k$ which satisfy $d \mu_{F}=\mu_{k}(1 \wedge d+d \wedge 1)$. If $F=k$ and $\pi=1_{F}$, we write $\operatorname{Der}_{R}^{*}(F)$ instead of $\operatorname{Der}_{R}^{*}(F, F)$. There is a natural $k_{*}$-homomorphism

$$
\psi: \operatorname{Der}_{R}^{*}(F, k) \rightarrow \operatorname{Hom}_{F_{*}}^{*}\left(I / I^{2}[1], k_{*}\right),
$$

defined by $\psi(d)(\bar{x})=\left(\mu_{k}\right)_{*} k_{*}^{R}(d)\left(\varphi_{F}^{k}(\bar{x})\right)$ for $d \in \operatorname{Der}_{R}^{*}(F, k)$ and $\bar{x} \in I / I^{2}[1]$. It is a homeomorphism if both $F$ and $k$ are regular quotient rings, where $\operatorname{Der}_{R}^{*}(F, k)$ is endowed with the subspace topology induced by the profinite topology on $k_{R}^{*}(F)$ and $\operatorname{Hom}_{F_{*}}^{*}\left(I / I^{2}[1], k_{*}\right) \cong D_{k_{*}}\left(k_{*} \otimes_{F_{*}} I / I^{2}[1]\right)$ with the dual-finite topology. The composition

$$
\operatorname{Hom}_{F_{*}}^{*}\left(I / I^{2}[1], k_{*}\right) \stackrel{\psi^{-1}}{\longrightarrow} \operatorname{Der}_{R}^{*}(F, k) \subseteq k_{R}^{*}(F)
$$

is independent of the products on $F$ and $k$. This result allows us to construct derivations. We restrict to the case where $k=F$ is a regular quotient ring and $\pi=1_{F}$ here. Let $\left(x_{1}, x_{2}, \ldots\right)$ be a regular sequence generating the ideal $I$ and let $\bar{x}_{i}^{\vee} \in D_{F_{*}}\left(I / I^{2}[1]\right)$ be dual to $\bar{x}_{i}$. The Bockstein operation $Q_{i} \in \operatorname{Der}_{R}^{*}(F)$ associated to $x_{i}$ is defined by $Q_{i}=\psi^{-1}\left(\bar{x}_{i}^{\vee}\right)$.

For a regular quotient ring $F$, the inclusion $\operatorname{Der}_{R}^{*}(F) \rightarrow F_{R}^{*}(F)$ lifts to a homeomorphism of $F^{*}$-algebras

$$
\widehat{\Lambda}\left(\operatorname{Der}_{R}^{*}(F)\right) \cong F_{R}^{*}(F),
$$

where $\widehat{\Lambda}\left(\mathscr{D e r} r_{R}^{*}(F)\right)$ denotes the completed exterior algebra on $\operatorname{Der}_{R}^{*}(F)$ and where $F_{R}^{*}(F)$ is endowed with the profinite topology. In particular we have $d^{2}=0$ for any $d \in \operatorname{Der}_{R}^{*}(F)$.

\section{The action of bilinear forms on products}

In this section, we show that there is a canonical action of the group of bilinear forms $\operatorname{Bil}\left(I / I^{2}[1]\right)$ on the set of products on a regular quotient $F=R / I$.

Let $F=R / I$ be a regular quotient and let $\operatorname{Prod}_{R}(F) \subseteq F_{R}^{*}(F \wedge F)$ denote the set of all products on $F$. Let $\operatorname{Per}\left(\operatorname{Prod}_{R}(F)\right)$ be the group of permutations of the $\operatorname{set} \operatorname{Prod}_{R}(F)$.

Writing $V$ for $I / I^{2}[1]$, we have a linear isomorphism [2, Lemma 6.15]

$$
\operatorname{Bil}(V)=D^{0}(V \otimes V) \cong(D(V) \hat{\otimes} D(V))^{0} .
$$


Composing it with the isomorphism

$$
(D(V) \hat{\otimes} D(V))^{0} \stackrel{\psi^{-1} \hat{\otimes} \psi^{-1}}{\longrightarrow}\left(\mathscr{D e r} r_{R}^{*}(F) \hat{\otimes} \mathscr{D} e r_{R}^{*}(F)\right)^{0}
$$

induced by the homeomorphism $\psi(2-4)$ yields an isomorphism of $F^{*}$-modules

$$
\operatorname{Bil}(V) \cong\left(\mathscr{D e r}_{R}^{*}(F) \hat{\otimes} \operatorname{Der}_{R}^{*}(F)\right)^{0} .
$$

The aim of this section is to prove the following result.

Proposition 3.1 Let $\mu \in \operatorname{Prod}_{R}(F)$ be a product and let $d, d^{\prime} \in \mathscr{D e r}_{R}^{*}(F)$ be derivations with $|d|=-\left|d^{\prime}\right|$. Then the composition

$$
\mu_{d, d^{\prime}}: F \wedge F \stackrel{1+d \wedge d^{\prime}}{\longrightarrow} F \wedge F \stackrel{\mu}{\rightarrow} F
$$

defines a product. This construction induces a group homomorphism

$$
\hat{\pi}:\left(\operatorname{Der}_{R}^{*}(F) \hat{\otimes} \operatorname{Der}_{R}^{*}(F)\right)^{0} \longrightarrow \operatorname{Per}\left(\operatorname{Prod}_{R}(F)\right),
$$

which gives rise via (3-1) to an action of $\operatorname{Bil}\left(I / I^{2}[1]\right)$ on $\operatorname{Prod}_{R}(F)$.

Notation 3.2 We refer to the action of Proposition 3.1 as the canonical action of $\operatorname{Bil}\left(I / I^{2}[1]\right)$ on $\operatorname{Prod}_{R}(F)$ in the sequel. The image of an element $(\beta, \mu) \in$ $\operatorname{Bil}\left(I / I^{2}[1]\right) \times \operatorname{Prod}_{R}(F)$ under the canonical action will be denoted by $\beta \mu$. Accordingly, $\beta F$ stands for $F$, endowed with the product $\beta \mu$.

Remark 3.3 The proof of Proposition 3.1 given below shows that in the special case $F=R / x$, the action of $\operatorname{Bil}\left((x) /(x)^{2}[1]\right) \cong F_{2|x|+2}$ coincides with the action defined in [9, Proposition 3.1].

Proof of Proposition 3.1 We first prove that $\bar{\mu}=\mu_{d, d^{\prime}}$ is associative, ie that we have $\bar{\mu}(1 \wedge \bar{\mu})=\bar{\mu}(\bar{\mu} \wedge 1)$. As a consequence of the isomorphism (2-5), derivations anticommute, ie for $d, d^{\prime} \in \operatorname{Der}_{R}^{*}(F)$, we have $d d^{\prime}=-d^{\prime} d$. Moreover, as a derivation, $d$ satisfies $d \mu=\mu(d \wedge 1+1 \wedge d)$. This yields

$$
\begin{aligned}
\bar{\mu}(\bar{\mu} \wedge 1)= & \mu\left(\left(\mu+\mu\left(d \wedge d^{\prime}\right)\right) \wedge 1\right)+\mu\left(d \wedge d^{\prime}\right)\left(\left(\mu+\mu\left(d \wedge d^{\prime}\right) \wedge 1\right)\right. \\
= & \mu(\mu \wedge 1)\left(1+d \wedge d^{\prime} \wedge 1\right)+\mu\left[(\mu(d \wedge 1+1 \wedge d)) \wedge d^{\prime}\right. \\
& \left.\quad+(\mu(d \wedge 1+1 \wedge d))\left(d \wedge d^{\prime}\right) \wedge d^{\prime}\right] \\
= & \mu(\mu \wedge 1)\left[1+d \wedge d^{\prime} \wedge 1+d \wedge 1 \wedge d^{\prime}+1 \wedge d \wedge d^{\prime}-d \wedge d d^{\prime} \wedge d^{\prime}\right]
\end{aligned}
$$


On the other hand, we obtain

$$
\begin{aligned}
& \bar{\mu}(1 \wedge \bar{\mu})=\mu\left(1 \wedge\left(\mu+\mu\left(d \wedge d^{\prime}\right)\right)\right)+\mu\left(d \wedge d^{\prime}\right)\left(1 \wedge\left(\mu+\mu\left(d \wedge d^{\prime}\right)\right)\right) \\
& =\mu(1 \wedge \mu)\left(1+1 \wedge d \wedge d^{\prime}\right)+\mu\left[d \wedge\left(\mu\left(d^{\prime} \wedge 1+1 \wedge d^{\prime}\right)\right)\right. \\
& \left.+d \wedge\left(\mu\left(d^{\prime} \wedge 1+1 \wedge d^{\prime}\right)\right)\left(d \wedge d^{\prime}\right)\right] \\
& =\mu(1 \wedge \mu)\left[1+1 \wedge d \wedge d^{\prime}+d \wedge d^{\prime} \wedge 1+d \wedge 1 \wedge d^{\prime}-d \wedge d d^{\prime} \wedge d^{\prime}\right],
\end{aligned}
$$

which proves that $\bar{\mu}$ is associative.

That $\bar{\mu}$ has $\eta_{F}: R \rightarrow F$ as a two-sided unit is an easy consequence of the fact that the composition $d \eta_{F}$ is trivial for a derivation $d$.

To prove that $\mu \mapsto \mu_{d, d^{\prime}}$ defines a permutation of $\operatorname{Prod}_{R}(F)$, it suffices to note that $\mu^{\prime} \mapsto \mu_{-d, d^{\prime}}^{\prime}$ is a two-sided inverse. This follows from

$$
\left(\mu_{d, d^{\prime}}\right)_{-d, d^{\prime}}=\left(\mu\left(1+d \wedge d^{\prime}\right)\right)\left(1-d \wedge d^{\prime}\right)=\mu .
$$

We have shown so far that $\left(d, d^{\prime}\right) \mapsto \mu_{d, d^{\prime}}$ defines a function

$$
\pi:\left(\operatorname{Der}_{R}^{*}(F) \times \operatorname{Der}_{R}^{*}(F)\right)^{0} \rightarrow \operatorname{Per}\left(\operatorname{Prod}_{R}(F)\right) .
$$

For derivations $d, d^{\prime}, e, e^{\prime}$ for which $|d|=-\left|d^{\prime}\right|$ and $|e|=-\left|e^{\prime}\right|$, we have that $\left(\mu_{d, d^{\prime}}\right)_{e, e^{\prime}}=\left(\mu_{e, e^{\prime}}\right)_{d, d^{\prime}}$ ie $\pi\left(e, e^{\prime}\right) \pi\left(d, d^{\prime}\right)=\pi\left(d, d^{\prime}\right) \pi\left(e, e^{\prime}\right)$. Using the two facts that (i) derivations square to zero and (ii) that $F$ is a quotient ring of $R$, one can check that $\pi$ is bilinear, ie $\pi\left(d, d^{\prime}+d^{\prime \prime}\right)=\pi\left(d, d^{\prime}\right) \pi\left(d, d^{\prime \prime}\right)$ and $\pi\left(d^{\prime}+d^{\prime \prime}, d\right)=$ $\pi\left(d^{\prime}, d\right) \pi\left(d^{\prime \prime}, d\right)$ for derivations $d, d^{\prime}, d^{\prime \prime}$ such that $\left|d^{\prime}\right|=\left|d^{\prime \prime}\right|=-|d|$. Hence $\pi$ induces a group homomorphism

$$
\bar{\pi}:\left(\operatorname{Der}_{R}^{*}(F) \otimes \operatorname{Der}_{R}^{*}(F)\right)^{0} \longrightarrow \operatorname{Per}\left(\operatorname{Prod}_{R}(F)\right) .
$$

Recall that $\operatorname{Der}_{R}^{*}(F)$ carries the topology inherited by the profinite topology on $F_{R}^{*}(F)$. We now show that $\bar{\pi}$ lifts to a group homomorphism

$$
\hat{\pi}:\left(\operatorname{Der}_{R}^{*}(F) \hat{\otimes} \operatorname{Der}_{R}^{*}(F)\right)^{0} \longrightarrow \operatorname{Per}\left(\operatorname{Prod}_{R}(F)\right) .
$$

Let $\operatorname{End}_{R}^{*}(F \wedge F)$ denote $(F \wedge F)_{R}^{*}(F \wedge F)$. Consider the homomorphism of monoids - with respect to addition and composition, respectively -

$$
\alpha:\left(\operatorname{Der}_{R}^{*}(F) \otimes \operatorname{Der}_{R}^{*}(F)\right)^{0} \rightarrow \operatorname{End}_{R}^{*}(F \wedge F),
$$

given by $\alpha\left(d \otimes d^{\prime}\right)=1+d \wedge d^{\prime}$. Observe that $\operatorname{End}_{R}^{*}(F \wedge F)$ is complete with respect to the profinite filtration, because the $(F \wedge F)_{*}$-module

$$
(F \wedge F)_{*}^{R}(F \wedge F) \cong(F \wedge F)_{*}^{R}(F) \otimes_{(F \wedge F)_{*}}(F \wedge F)_{*}^{R}(F)
$$


is free (compare [5, Remark 2.23]). Composition $\circ$ in $\operatorname{End}_{R}^{*}(F \wedge F)$ is clearly continuous, and so $\left(\operatorname{End}_{R}^{*}(F \wedge F), \circ\right)$ is a complete topological monoid. It is easily checked that $\alpha$ is a continuous homomorphism of topological monoids. Moreover, the action of $\left(\mathscr{D e r} r_{R}^{*}(F) \otimes \operatorname{Der}_{R}^{*}(F)\right)^{0}$ on $\operatorname{Prod}_{R}(F)$ induced by $\bar{\pi}$ is compatible with the canonical right action of $\operatorname{End}_{R}^{*}(F \wedge F)$ on $F_{R}^{*}(F \wedge F)$ via $\alpha$. Since $\operatorname{End}_{R}^{*}(F \wedge F)$ is complete, $\alpha$ lifts to a continuous homomorphism

$$
\left.\hat{\alpha}: \operatorname{Der}_{R}^{*}(F) \hat{\otimes} \operatorname{Der}_{R}^{*}(F)\right)^{0} \rightarrow \operatorname{End}_{R}^{*}(F \wedge F) .
$$

For the construction of $\hat{\pi}$, it remains to show that this action restricts to an action on $\operatorname{Prod}_{R}(F)$. For this, we use the facts that (i) the action of $\operatorname{End}_{R}^{*}(F \wedge F)$ on $F_{R}^{*}(F \wedge F)$ is continuous and (ii) that $\operatorname{Prod}_{R}(F)$ is closed in $F_{R}^{*}(F \wedge F)$. The verification of Fact (i) is easy. To prove (ii), we consider

$$
a: F_{R}^{*}(F \wedge F) \longrightarrow F_{R}^{*}(F \wedge F \wedge F), a(f)=f(f \wedge 1)-f(1 \wedge f),
$$

and the homomorphisms

$$
l, r: F_{R}^{*}(F \wedge F) \longrightarrow F_{R}^{*}(F), l(f)=f\left(1 \wedge \eta_{F}\right), r(f)=f\left(\eta_{F} \wedge 1\right),
$$

where we implicitly use the equivalences $R \wedge F \simeq F \simeq F \wedge R$. Observe that

$$
\operatorname{Prod}_{R}(F)=\operatorname{ker}(a) \cap \operatorname{ker}(l) \cap \operatorname{ker}(r) \cap F_{R}^{0}(F \wedge F) \subseteq F_{R}^{*}(F \wedge F) .
$$

Because $a, l$ and $r$ are continuous and because their targets are Hausdorff, their kernels are closed. Moreover, so is $F_{R}^{0}(F \wedge F)$ and hence $\operatorname{Prod}_{R}(F)$.

It follows that $\operatorname{Prod}_{R}(F)$ is complete, as a closed subset of the complete module $F_{R}^{*}(F \wedge F)$. This implies that the action of $\left(\mathscr{D e r} r_{R}^{*}(F) \hat{\otimes} \operatorname{Der}_{R}^{*}(F)\right)^{0}$ on $F_{R}^{*}(F \wedge F)$ restricts to an action on $\operatorname{Prod}_{R}(F)$, and we are done.

\section{Classification of products}

In this section, we show that the action of bilinear forms on $I / I^{2}[1]$ on the set of products on a regular quotient $F=R / I$ classify the products on $F$. The main result is the following theorem.

Theorem 4.1 Let $F=R / I$ be a regular quotient. Then the canonical action of the group of bilinear forms $\operatorname{Bil}\left(I / I^{2}[1]\right)$ on the set of products $\operatorname{Prod}_{R}(F)$ is free and transitive.

The strategy for the proof is as follows. On fixing "coordinates", we first give an explicit formula for $\beta \mu$, for $\beta \in \operatorname{Bil}\left(I / I^{2}[1]\right)$ and $\mu \in \operatorname{Prod}_{R}(F)$ (Lemma 4.2). Secondly, 
we give an explicit description of all products on $F$ (Lemma 4.3). With these two ingredients, we prove Theorem 4.1.

We first fix some notation. Let $F=R / I$ be a regular quotient and let $\mu \in \operatorname{Prod}_{R}(F)$ be an arbitrary fixed product on $F$ (such a $\mu$ always exists - see [9, Corollary 2.10; 5]). Let $\left(x_{1}, x_{2}, \ldots\right)$ be a regular sequence generating $I$. Then the residue classes $\bar{x}_{i} \in$ $V=I / I^{2}[1]$ form a basis, and we let $\bar{x}_{i}^{\vee} \in D(V)$ denote the dual elements. An arbitrary bilinear form $\beta \in \operatorname{Bil}(V)$ can be uniquely written as a (possibly infinite) sum $\beta=\sum v_{i j} \bar{x}_{i}^{\vee} \otimes \bar{x}_{j}^{\vee}$, with $v_{i j}=\beta\left(\bar{x}_{i} \otimes \bar{x}_{j}\right) \in F_{*}$. Recall that $\psi: \operatorname{Der}_{R}^{*}(F) \rightarrow D(V)$ from (2-4) maps the Bockstein operation $Q_{i}$ to $\bar{x}_{i}^{\vee}$, by definition of $Q_{i}$.

Now it is easy to check $\left(\prod_{i+j \leqslant k}\left(1+v_{i j} Q_{i} \wedge Q_{j}\right)\right)_{k}$ is a Cauchy sequence in the complete $F_{*}$-module $\operatorname{End}_{R}^{*}(F \wedge F)($ compare Section 3$)$. We define $\prod_{i, j}\left(1+v_{i j} Q_{i} \wedge Q_{j}\right)$ to be its limit.

By definition of the canonical action of $\operatorname{Bil}(V)$ on $\operatorname{Prod}_{R}(F)$, we have:

Lemma 4.2 In the notation from above, the product $\beta \mu$ is given by

$$
\beta \mu=\mu \circ \prod_{i, j}\left(1+v_{i j} Q_{i} \wedge Q_{j}\right) .
$$

The next lemma describes the set of all products on $F$. It appears in Angelveit [1, Theorem 3.9]. We provide a complete proof here. It makes essential use of the existence of the canonical action of $\operatorname{Bil}\left(I / I^{2}[1]\right)$ on $\operatorname{Prod}_{R}(F)$, which in turn relies crucially on the fact proved in [5] that $\operatorname{Der}_{R}^{*}(F)$ is independent of the product on $F$, as a submodule of $F_{R}^{*}(F)$.

Lemma 4.3 For any product $\bar{\mu} \in \operatorname{Prod}_{R}(F)$, there exist uniquely determined elements $v_{i j} \in F_{*}$ of degree $\left|v_{i j}\right|=\left|Q_{i}\right|+\left|Q_{j}\right|$ such that

$$
\bar{\mu}=\mu \circ \prod_{i, j}\left(1+v_{i j} Q_{i} \wedge Q_{j}\right) .
$$

The proof of Lemma 4.3 is postponed to the end of the section. We first prove Theorem 4.1 .

Proof of Theorem 4.1 To prove transitivity, let $\mu, \bar{\mu} \in \operatorname{Prod}_{R}(F)$ be arbitrary products. According to Lemma 4.3, we can write $\bar{\mu}$ as

$$
\bar{\mu}=\mu \circ \prod\left(1+v_{i j} Q_{i} \wedge Q_{j}\right) .
$$

On setting $\beta=\sum v_{i j} \bar{x}_{i}^{\vee} \otimes \bar{x}_{j}^{\vee}$, we obtain $\beta \mu=\bar{\mu}$, by Lemma 4.2.

Freeness of the action follows from the fact that the coefficients $v_{i j}$ in Lemma 4.3 are uniquely determined. 
We need some notation for the proof of Lemma 4.3. Let $a_{i} \in F_{*}^{R}(F)$ be the image of the residue class $\bar{x}_{i} \in V$ under the characteristic homomorphism $\varphi: V \rightarrow F_{*}^{R}(F)$. By (2-3), we have $\left(F^{\mathrm{op}}\right)_{*}^{R}(F) \cong \Lambda\left(a_{1}, a_{2}, \ldots\right)$. Under this isomorphism, $\left(F^{\mathrm{op}}\right)_{*}\left(Q_{i}\right)$ corresponds to the partial derivative $\partial / \partial a_{i}$; see [5, Remark 4.5]. For a multi-index $I=\left(i_{1}, \ldots, i_{m}\right)$ with $i_{1}<\cdots<i_{m}$, we write $|I|$ for $m, Q_{I}$ for $Q_{i_{1}} \cdots Q_{i_{m}}$ and $a_{I}$ for $a_{i_{1}} \wedge \cdots \wedge a_{i_{m}}$.

Proof of Lemma 4.3 The Künneth homeomorphism (see [10, Section 2])

$$
\kappa: F_{R}^{*}(F) \hat{\otimes} F_{R}^{*}(F) \stackrel{\cong}{\longrightarrow} F_{R}^{*}(F \wedge F)
$$

maps $\sum x_{I J} Q_{I} \otimes Q_{J}$ to $\mu \circ\left(\sum x_{I J} Q_{I} \wedge Q_{J}\right)$. Since $\bar{\mu} \in F_{R}^{0}(F \wedge F)$, we may write $\bar{\mu}=\mu \circ\left(\sum w_{I J} Q_{I} \wedge Q_{J}\right)$, with $\left|w_{I J}\right|=\left|Q_{I}\right|+\left|Q_{J}\right|$. In particular, $w_{I J} \neq 0$ only for $|I|+|J|$ even. Since $\bar{\mu}$ has a two sided unit, it follows that $w_{\varnothing J}=w_{I \varnothing}=0$ for all $I, J$. Hence $\bar{\mu}$ can be written as

$$
\bar{\mu}=\mu \circ\left(1+\sum_{|I|,|J|>0} w_{I J} Q_{I} \wedge Q_{J}\right)=\kappa\left(1+\sum_{|I|,|J|>0} w_{I J} Q_{I} \otimes Q_{J}\right) .
$$

In a first step, we show that there exist $v_{I J} \in F_{*}$ such that

$$
1+\sum_{|I|,|J|>0} w_{I J} Q_{I} \wedge Q_{J}=\prod_{|I|,|J|>0}\left(1+v_{I J} Q_{I} \wedge Q_{J}\right)
$$

where the product is taken in the monoid $\operatorname{End}_{R}^{*}(F \wedge F)$.

If $\left(x_{1}, x_{2}, \ldots\right)$ is finite, the sum on the left hand side of (4-1) is of the form

$$
1+\sum_{k=1}^{n} w_{I_{k} J_{k}} Q_{I_{k}} \wedge Q_{J_{k}}
$$

Set $\alpha_{k}=w_{I_{k} J_{k}} Q_{I_{k}} \wedge Q_{J_{k}}$ and observe that $\alpha_{k}^{2}=0$. A tedious computation shows that there exist coefficients $c_{i_{1} \cdots i_{k}} \in F_{*}$ such that

$$
\prod_{\substack{k=1, \ldots, n \\ 1 \leqslant i_{1}<\cdots<i_{k} \leqslant n}}\left(1+c_{i_{1} \cdots i_{k}} \alpha_{i_{1}} \cdots \alpha_{i_{k}}\right)=1+\sum_{k=1}^{n} \alpha_{k}
$$

This shows (4-1) for finite sequences $\left(x_{1}, x_{2}, \ldots\right)$. The general case follows from this by passing to limits. 
In a second step, we use the associativity of $\bar{\mu}$ to show that the coefficients $v_{I J}$ in (4-1) are zero for $|I|+|J|>2$. We write

$$
\bar{\mu}=\mu \circ \prod_{i, j}\left(1+v_{i j} Q_{i} \wedge Q_{j}\right) \circ \prod_{|I|+|J|>2}\left(1+v_{I J} Q_{I} \wedge Q_{J}\right)
$$

and let $\beta=\sum\left(-v_{i j}\right) \bar{x}_{i}^{\vee} \otimes \bar{x}_{j}^{\vee} \in \operatorname{Bil}(V)$. From Lemma 4.2, we deduce

$$
\beta \bar{\mu}=\mu \circ \prod_{|I|+|J|>2}\left(1+v_{I J} Q_{I} \wedge Q_{J}\right) .
$$

We set $\tilde{\mu}=\beta \bar{\mu} \in \operatorname{Prod}_{R}(F)$ and assume that $\mathcal{I}=\left\{(I, J) \mid v_{I J} \neq 0\right\}$ is nonempty. We will show below that this implies that the two morphisms

$$
\tilde{\mu}_{*}\left(\tilde{\mu}_{*} \otimes 1\right), \tilde{\mu}_{*}\left(1 \otimes \tilde{\mu}_{*}\right):\left(F^{\mathrm{op}}\right)_{*}^{R}(F)^{\otimes 3} \longrightarrow\left(F^{\mathrm{op}}\right)_{*}^{R}(F)
$$

are different, where $\tilde{\mu}_{*}$ stands for $m_{\widetilde{F}}^{F^{\text {op }}}$. It follows that $\tilde{\mu}$ is not associative, which is a contradiction. Therefore, $\mathcal{I}$ is empty, and the statement is proved.

Let $\left(I_{0}, J_{0}\right) \in \mathcal{I}$ such that $\left|I_{0}\right|+\left|J_{0}\right|$ is minimal. In the case where $\left|I_{0}\right|>1$, we set $I_{0}=(L, M)$ with $|L|,|M| \geqslant 1$. If $\left|I_{0}\right|=1$, we decompose $J_{0}$ in the same way. We show that the two morphisms of (4-3) don't agree by evaluating them on $a_{L} \otimes a_{M} \otimes a_{J_{0}}$ if $\left|I_{0}\right|>1$ or on $a_{I_{0}} \otimes a_{L} \otimes a_{M}$ if $\left|I_{0}\right|=1$.

As $\left(F^{\mathrm{op}}\right)_{*}^{R}(F) \cong \Lambda\left(a_{1}, a_{2}, \ldots\right)$, the set of elements $\left\{a_{I} \otimes a_{J} \otimes a_{K}\right\}_{I, J, K}$ forms a basis of the free $F_{*}$-module $\left(F^{\mathrm{op}}\right)_{*}^{R}(F)^{\otimes 3}$. By minimality of $\left(I_{0}, J_{0}\right)$, we have $|I|>|L|$ or $|J|>|M|$ for any $(I, J) \in \mathcal{I}$. This shows that

$$
F_{*}\left(Q_{I}\right) \otimes F_{*}\left(Q_{J}\right)\left(a_{L} \otimes a_{M}\right)=0
$$

for all $(I, J) \in \mathcal{I}$. For $a, b \in F_{*}^{R}\left(F^{\text {op }}\right)$, let us write $a \wedge b$ for $m_{F}^{F^{\text {op }}}(a \otimes b)$. Using (4-4), we find

$$
\begin{aligned}
\tilde{\mu}_{*}\left(\tilde{\mu}_{*} \otimes 1\right)\left(a_{L} \otimes a_{M} \otimes a_{J_{0}}\right) & =\tilde{\mu}_{*}\left(\mu_{*} \otimes 1\right)\left(a_{L} \otimes a_{M} \otimes a_{J_{0}}\right) \\
& =\tilde{\mu}_{*}\left(a_{L} \wedge a_{M} \otimes a_{J_{0}}\right) \\
& =\tilde{\mu}_{*}\left(a_{I_{0}} \otimes a_{J_{0}}\right) \\
& =\mu_{*}\left(a_{I_{0}} \otimes a_{J_{0}}-v_{I_{0} J_{0}} \cdot 1 \otimes 1\right)=a_{\left(I_{0}, J_{0}\right)}-v_{I_{0} J_{0}} \cdot 1 .
\end{aligned}
$$

(The negative sign appears because the elements involved have odd degree.) Similarly, $\tilde{\mu}_{*}\left(1 \otimes \tilde{\mu}_{*}\right)\left(a_{L} \otimes a_{M} \otimes a_{J_{0}}\right)=\tilde{\mu}_{*}\left(1 \otimes \mu_{*}\right)\left(a_{L} \otimes a_{M} \otimes a_{J_{0}}\right)$

$$
\begin{aligned}
& =\tilde{\mu}_{*}\left(a_{L} \otimes a_{M} \wedge a_{J_{0}}\right) \\
& =\tilde{\mu}_{*}\left(a_{L} \otimes a_{\left(M, J_{0}\right)}\right)=\mu_{*}\left(a_{L} \otimes a_{\left(M, J_{0}\right)}\right)=a_{\left(I_{0}, J_{0}\right)} .
\end{aligned}
$$

This shows that the two morphisms in (4-3) are different, as required. 
Uniqueness of the coefficients $v_{i j}$ follows from the equality

$$
\tilde{\mu}_{*}\left(a_{i} \otimes a_{j}\right)=a_{i} \wedge a_{j}-v_{i j} \cdot 1,
$$

which we used in the argument above. This concludes the proof.

\section{Transformation rules for the characteristic bilinear form}

In this section, we describe how the action of the bilinear forms affects characteristic bilinear forms and draw some consequences.

Proposition 5.1 Let $(F, k, \pi)$ be an admissible pair, where $F=R / I$ is a regular quotient ring. For a bilinear form $\beta \in \operatorname{Bil}\left(I / I^{2}[1]\right)$, we have

$$
b_{\beta F}^{k}=b_{F}^{k}-k_{*} \otimes \beta \text {. }
$$

Proposition 5.2 Let $\left(F, \bar{F}, 1_{F}\right)$ be an admissible pair, where $F, \bar{F}$ are regular quotient rings with the same underlying quotient module $R / I$, endowed with two (possibly) different products. For $\beta \in \operatorname{Bil}\left(I / I^{2}[1]\right)$, we have

$$
b_{F}^{\beta \bar{F}}=b_{F}^{\bar{F}}-\beta^{t} .
$$

The proof of these two propositions is technical and will be given at the end of this section. We draw some consequences first.

Corollary 5.3 Let $b_{F}$ be the characteristic bilinear form of a regular quotient ring $F=R / I$ and let $\beta \in \operatorname{Bil}\left(I / I^{2}[1]\right)$ be a bilinear form. Then the characteristic bilinear form of $\beta F$ is given by

$$
b_{\beta F}=b_{F}-\left(\beta+\beta^{t}\right) .
$$

Proof The equalities of Propositions 5.1 and 5.2 imply that

$$
b_{\beta F}=b_{\beta F}^{\beta F}=b_{F}^{\beta F}-\beta=b_{F}^{F}-\beta^{t}-\beta=b_{F}-\left(\beta+\beta^{t}\right) .
$$

Corollary 5.4 The characteristic bilinear form $b_{F}$ of a regular quotient ring $F$ is symmetric.

Proof Let $\mu$ denote the product on $F=R / I$. By [9, Corollary 2.10; 5], there exists a diagonal product $\bar{\mu}$ on $F$ with respect to some regular sequence $\left(x_{1}, x_{2}, \ldots\right)$ generating $I$. By Theorem 4.1, there exists $\beta \in \operatorname{Bil}\left(I / I^{2}[1]\right)$ with $\beta \bar{F}=F$. Corollary 5.3 implies that $b_{F}=b_{\bar{F}}-\left(\beta+\beta^{t}\right)$. Now $b_{\bar{F}}$ is diagonal with respect to the basis $\bar{x}_{1}, \bar{x}_{2}, \ldots$ of $I / I^{2}[1]$ associated to the sequence $\left(x_{1}, x_{2}, \ldots\right)$ [5, Proposition 2.35]. Therefore, $b_{F}$ is the sum of two symmetric bilinear forms and therefore symmetric. 
Corollary 5.5 For a regular quotient ring $F$ with characteristic bilinear form $b_{F}$, we have $F^{\mathrm{op}}=b_{F} F$ and $b_{F}$ op $=-b_{F}$. Therefore, $F$ is commutative if and only if $b_{F}=0$.

Proof As the bilinear forms $\operatorname{Bil}\left(I / I^{2}[1]\right)$ act transitively on $\operatorname{Prod}_{R}(F)$, there exists $\beta \in \operatorname{Bil}\left(I / I^{2}[1]\right)$ with $F^{\text {op }}=\beta F$. Proposition 5.1 implies that $b_{F \text { op }}^{F}=b_{\beta F}^{F}=b_{F}-\beta$. But $b_{F \text { op }}^{F}$ is trivial by [5, Proposition 2.21] and so $\beta=b_{F}$. From Corollary 5.3, we deduce that $b_{F \text { op }}=b_{F}-\left(b_{F}+b_{F}^{t}\right)=-b_{F}$, since $b_{F}$ is symmetric.

Remark 5.6 Let $(F=R / I, \mu)$ be a regular quotient ring which is diagonal with respect to some regular sequence $\left(x_{1}, x_{2}, \ldots\right)$ generating $I$. Then $b_{F} \in \operatorname{Bil}\left(I / I^{2}[1]\right)$ is diagonal with respect to the basis $\bar{x}_{1}, \bar{x}_{2}, \ldots$, as we used above. Thus $b_{F}$ can be written as $\sum \alpha_{i} \bar{x}_{i}^{\vee} \otimes \bar{x}_{i}^{\vee}$, where $\alpha_{i} \in F_{*}$ and where $\bar{x}_{i}^{\vee}$ denotes the dual of $\bar{x}_{i}$. From Corollary 5.5, we obtain

$$
\mu^{\mathrm{op}}=b_{F} \mu=\mu \circ \prod_{i}\left(1+\alpha_{i} Q_{i} \wedge Q_{i}\right)
$$

where $Q_{i}$ denotes the Bockstein operation associated to $\bar{x}_{i}^{\vee}$. This generalizes wellknown formulas for $P(n)$ and $K(n)$ (see Section 8).

We now proceed to the proofs of Proposition 5.1 and Proposition 5.2.

Observe that it suffices to verify the statements for bilinear forms $\beta$ of the form $\beta=\alpha \otimes \alpha^{\prime}$ with $\alpha, \alpha^{\prime} \in D\left(I / I^{2}[1]\right)$, because an arbitrary bilinear form can be written as a (possibly infinite) sum of bilinear forms of this type.

We first fix some notation used for the proofs. The proof of each proposition is then preceded by a lemma.

Let $(F, k, \pi)$ be an admissible pair. For the proof of Proposition 5.2, $k$ will be $\bar{F}$ and $\pi=1_{F}$. Let $\mu$ denote the product on $F$ and $v$ the one on $k$. For $k=\bar{F}$, we write $\bar{\mu}$ instead of $v$, as usual. We let $V=I / I^{2}[1]$ and consider $\beta=\alpha \otimes \alpha^{\prime} \in \operatorname{Bil}(V)$, where $\alpha, \alpha^{\prime} \in D(V)$. We let $d, d^{\prime} \in \operatorname{Der}_{R}^{*}(F)$ be the derivations corresponding under $\psi: \operatorname{Der}_{R}^{*}(F) \cong D(V)$ to $\alpha, \alpha^{\prime}$, respectively. By definition of the action of $\operatorname{Bil}(V)$ on $\operatorname{Prod}_{R}(F)$, we have (using notation from Section 3)

$$
\beta \mu=\left(\alpha \otimes \alpha^{\prime}\right) \mu=\mu_{d, d^{\prime}}=\mu\left(1+d \wedge d^{\prime}\right) .
$$

We write $\bar{x}, \bar{y}$ for the residue classes of elements $x, y \in I$ in both $V$ and in $k_{*} \otimes_{F_{*}} V$. Recall that $b_{F}^{k}$ is defined as $b_{F}^{k}(\bar{x} \otimes \bar{y})=v_{*} k_{*}(\pi)(\varphi(\bar{x}) \cdot \varphi(\bar{y}))$, where $\varphi$ is the characteristic homomorphism $\varphi_{F}^{k}: V \rightarrow k_{*}^{R}(F)$ and where $a \cdot b=m_{F}^{k}(a \otimes b) \in k_{*}^{R}(F)$ for $a, b \in k_{*}^{R}(F)$ given in (2-2). 
Lemma 5.7 Let $(F, k, \pi)$ be an admissible pair, where $F=R / I$ is a regular quotient ring. For $\beta$ a bilinear form in $\operatorname{Bil}\left(I / I^{2}[1]\right)$ and $x, y \in I$, we have

$$
m_{\beta F}^{k}(\varphi(\bar{x}) \otimes \varphi(\bar{y}))=\varphi(\bar{x}) \cdot \varphi(\bar{y})-\pi_{*}(\beta(\bar{x} \otimes \bar{y})) \cdot 1 .
$$

Proof Let $\beta=\alpha \otimes \alpha^{\prime}$ with $\alpha, \alpha^{\prime} \in D(V)$. Recall the definition of $m_{\beta F}^{k}$ :

$$
m_{\beta F}^{k}(\varphi(\bar{x}) \otimes \varphi(\bar{y}))=(\nu \wedge \beta \mu)_{*}(1 \wedge \tau \wedge 1)_{*} \zeta(\varphi(\bar{x}) \otimes \varphi(\bar{y})),
$$

where $\zeta: k_{*}^{R}(F) \otimes k_{*}^{R}(F) \rightarrow(k \wedge F \wedge k \wedge F)_{*}$ is the canonical map and $\tau$ the switch map $\tau: F \wedge k \rightarrow k \wedge F$. From the definition of $\beta \mu$, we deduce that

$$
\begin{aligned}
m_{\beta F}^{k}(\varphi(\bar{x}) & \otimes \varphi(\bar{y})) \\
& =\left(v \wedge \mu+v \wedge\left(\mu \circ d \wedge d^{\prime}\right)\right)_{*}(1 \wedge \tau \wedge 1)_{*} \zeta(\varphi(\bar{x}) \otimes \varphi(\bar{y})) \\
& =\varphi(\bar{x}) \cdot \varphi(\bar{y})-(v \wedge \mu)_{*}(1 \wedge \tau \wedge 1)_{*} \zeta\left((1 \wedge d)_{*}(\varphi(\bar{x})) \otimes\left(1 \wedge d^{\prime}\right)_{*}(\varphi(\bar{y}))\right) \\
& =\varphi(\bar{x}) \cdot \varphi(\bar{y})-(v \wedge \mu)_{*}(1 \wedge \tau \wedge 1)_{*} \zeta\left(k_{*}^{R}(d)(\varphi(\bar{x})) \otimes k_{*}^{R}\left(d^{\prime}\right)(\varphi(\bar{y}))\right) .
\end{aligned}
$$

By [5, Lemma 4.11], we have that $k_{*}^{R}(d)(\varphi(\bar{x}))=\alpha(\bar{x}) \cdot 1$ and $k_{*}^{R}\left(d^{\prime}\right)(\varphi(\bar{y}))=\alpha^{\prime}(\bar{y}) \cdot 1$, which implies the statement.

Proof of Proposition 5.1 Let $\beta=\alpha \otimes \alpha^{\prime}, \alpha, \alpha^{\prime} \in D(V)$. Lemma 5.7 implies

$$
\begin{aligned}
b_{\beta F}^{k}(\bar{x} \otimes \bar{y}) & =v_{*}\left(m_{\beta F}^{k}(\varphi(\bar{x}) \otimes \varphi(\bar{y}))\right)=v_{*}\left(\varphi(\bar{x}) \cdot \varphi(\bar{y})-\alpha(\bar{x}) \alpha^{\prime}(\bar{y}) \cdot 1\right) \\
& =b_{F}^{k}(\bar{x} \otimes \bar{y})-\alpha(\bar{x}) \alpha^{\prime}(\bar{y}) .
\end{aligned}
$$

Lemma 5.8 For $F, \bar{F}$ as in Proposition 5.2 and $\beta \in \operatorname{Bil}\left(I / I^{2}[1]\right)$, we have

$$
m_{F}^{\beta \bar{F}}(\varphi(\bar{x}) \otimes \varphi(\bar{y}))=\varphi(\bar{x}) \cdot \varphi(\bar{y})-\beta(\bar{x} \otimes \bar{y}) \cdot 1 .
$$

Proof Let $\beta=\alpha \otimes \alpha^{\prime}$ with $\alpha, \alpha^{\prime} \in D(V)$. By definition of $\beta \bar{\mu}$, we have $m_{F}^{\beta \bar{F}}(\varphi(\bar{x}) \otimes \varphi(\bar{y}))$

$$
\begin{aligned}
& =\left(\bar{\mu} \wedge \mu+\left(\bar{\mu} \circ d \wedge d^{\prime}\right) \wedge \mu\right)_{*}(1 \wedge \tau \wedge 1)_{*} \zeta(\varphi(\bar{x}) \otimes \varphi(\bar{y})) \\
& =\varphi(\bar{x}) \cdot \varphi(\bar{y})+(\bar{\mu} \wedge \mu)_{*}(1 \wedge \tau \wedge 1)_{*} \zeta\left((d \wedge 1)_{*} \otimes\left(d^{\prime} \wedge 1\right)_{*}(\varphi(\bar{x}) \otimes \varphi(\bar{y}))\right) \\
& =\varphi(\bar{x}) \cdot \varphi(\bar{y})-(\bar{\mu} \wedge \mu)_{*}(1 \wedge \tau \wedge 1)_{*} \zeta\left((d \wedge 1)_{*}(\varphi(\bar{x})) \otimes\left(d^{\prime} \wedge 1\right)_{*}(\varphi(\bar{y}))\right) .
\end{aligned}
$$

It remains to identify the second summand of the last equality above. By definition, we have $(1 \wedge d)_{*}=F_{*}^{R}(d)$, and furthermore

$$
(d \wedge 1)_{*}=\tau_{*}(1 \wedge d)_{*} \tau_{*}=\tau_{*} F_{*}^{R}(d) \tau_{*} .
$$


From [5, Proposition 3.6], we obtain $\tau_{*} \varphi(\bar{x})=-\varphi(\bar{x})$. By [5, Lemma 4.11], we have $F_{*}^{R}(d)(\varphi(\bar{x}))=\alpha(\bar{x}) \cdot 1$. This yields $(d \wedge 1)_{*}(\varphi(\bar{x}))=-\alpha(\bar{x}) \cdot 1$. Analogously, we obtain $\left(d^{\prime} \wedge 1\right)_{*}(\varphi(\bar{y}))=-\alpha^{\prime}(\bar{y}) \cdot 1$, and we are done.

Proof of Proposition 5.2 For $\beta=\alpha \otimes \alpha^{\prime}$ with $\alpha, \alpha^{\prime} \in D(V)$, we compute

$$
\begin{aligned}
b_{F}^{\beta \bar{F}}(\bar{x} \otimes \bar{y}) & =(\beta \bar{\mu})_{*}\left(m_{F}^{\beta \bar{F}}(\varphi(\bar{x}) \otimes \varphi(\bar{y}))\right)=(\beta \bar{\mu})_{*}\left(\varphi(\bar{x}) \cdot \varphi(\bar{y})-\alpha(\bar{x}) \alpha^{\prime}(\bar{y}) \cdot 1\right) \\
& \left.=\left(\bar{\mu}+\bar{\mu}\left(d \wedge d^{\prime}\right)\right)_{*}(\varphi(\bar{x}) \cdot \varphi(\bar{y}))-\alpha(\bar{x}) \alpha^{\prime}(\bar{y}) \cdot 1\right) \\
& \left.=b_{\mu}^{\bar{\mu}}(\bar{x} \otimes \bar{y})-\alpha(\bar{x}) \alpha^{\prime}(\bar{y})+\left(\bar{\mu}\left(d \wedge d^{\prime}\right)\right)_{*}(\varphi(\bar{x}) \cdot \varphi(\bar{y}))\right) .
\end{aligned}
$$

The first equality holds by definition of the characteristic bilinear form, the second by Lemma 5.7, the third by definition of $\beta \bar{\mu}$ and the fourth because $d$ and $d^{\prime}$ are derivations and so are trivial on 1 .

Since $\alpha(\bar{x}) \alpha^{\prime}(\bar{y})=\beta(\bar{x} \otimes \bar{y})$, it remains to show that

$$
\left(\mu\left(d \wedge d^{\prime}\right)\right)_{*}(\varphi(\bar{x}) \cdot \varphi(\bar{y}))=\beta(\bar{x} \otimes \bar{y})-\beta^{t}(\bar{x} \otimes \bar{y}) .
$$

To prove this, we write $d \wedge d^{\prime}$ as $(d \wedge 1)\left(1 \wedge d^{\prime}\right)$. Using computations from the proof of Lemma 5.8 and the fact that $F_{*}^{R}(d)$ and $F_{*}^{R}\left(d^{\prime}\right)$ are derivations with respect to $m_{F}^{\bar{F}}$ (see [5, Lemma 4.3]), we obtain

$$
\begin{aligned}
\left(\mu\left(d \wedge d^{\prime}\right)\right)_{*}(\varphi(\bar{x}) \cdot \varphi(\bar{y})) & =\bar{\mu}_{*}(d \wedge 1)_{*}\left(1 \wedge d^{\prime}\right)_{*}(\varphi(\bar{x}) \cdot \varphi(\bar{y})) \\
& =\bar{\mu}_{*}(d \wedge 1)_{*}\left(\alpha^{\prime}(\bar{x}) \varphi(\bar{y})-\varphi(\bar{x}) \alpha^{\prime}(\bar{y})\right) \\
& =\bar{\mu}_{*}\left(-\alpha^{\prime}(\bar{x}) \alpha(\bar{y}) \cdot 1+\alpha(\bar{x}) \alpha^{\prime}(\bar{y}) \cdot 1\right) \\
& =\left(\alpha \otimes \alpha^{\prime}\right)(\bar{x} \otimes \bar{y}-\bar{y} \otimes \bar{x})=\beta(\bar{x} \otimes \bar{y})-\beta^{t}(\bar{x} \otimes \bar{y}),
\end{aligned}
$$

and the proposition is proven.

\section{Maps of quotient ring spectra}

In this section, we determine which maps $\pi: F \rightarrow G$ between regular quotient rings are multiplicative. We start with a definition.

Definition 6.1 An admissible pair $(F, G, \pi)$ with $F=R / I$ and $G=R / J$ is called smooth if the canonical homomorphism $\pi_{*}: G_{*} \otimes_{F_{*}} I / I^{2}[1] \rightarrow J / J^{2}[1]$ is split injective, ie $\pi_{*}$ is injective and its image in $J / J^{2}[1]$ is a direct summand. If there is no risk of confusion, we say that $I \subseteq J$ is smooth.

Theorem 6.2 Let $(F, G, \pi)$ be an admissible pair for which $F=R / I$ and $G=R / J$ are regular quotient rings and which is smooth. Then $\pi$ is multiplicative if and only if $G_{*} \otimes b_{F}=b_{F}^{G}=\pi^{*}\left(b_{G}\right)$. 
The strategy for the proof is as follows. We first prove the result in the special case where $F$ is diagonal. As in this case the smoothness hypothesis is unnecessary, we formulate a separate statement (Proposition 6.3). After assembling some auxiliary results (Lemma 6.5 and Lemma 6.6), we prove Theorem 6.2 by reducing it to the case where $F$ is diagonal.

Proposition 6.3 Let $(F, G, \pi)$ be an admissible pair for which $F=R / I$ and $G=$ $R / J$ are regular quotient rings. Assume that $F$ is diagonal. Then $\pi$ is multiplicative if and only if $G_{*} \otimes b_{F}=b_{F}^{G}=\pi^{*}\left(b_{G}\right)$.

Proof If $\pi$ is multiplicative, $(F, G, \pi)$ is a multiplicative admissible pair by definition and the assertion follows from [5, Proposition 2.20].

To prove the converse, fix a regular sequence $\left(x_{1}, x_{2}, \ldots\right)$ generating $I$, for which there are products $\mu_{k}$ on the $R / x_{k}$ such that the product $\mu_{F}$ on $F$ is the smash product of the $\mu_{k}$. Let $\pi_{k}$ stand for the composition $\pi j_{k}: R / x_{k} \rightarrow F \rightarrow G$, where $j_{k}: R / x_{k} \rightarrow F$ is the canonical map.

By [9, Proposition 4.8], the map $\pi: F \rightarrow G$ is multiplicative if and only if (i) all the $\pi_{k}$ are multiplicative and (ii) $\pi_{k}$ commutes with $\pi_{l}$ for $k \neq l$.

In a first step, we show that the $\pi_{k}$ are multiplicative, ie that they satisfy $\mu_{G}\left(\pi_{k} \wedge \pi_{k}\right)=$ $\pi_{k} \mu_{k}$, where $\mu_{G}$ is the product on $G$. Because $x_{k} \in I \subseteq J$, the $G_{*}$-module $G_{*}^{R}\left(R / x_{k}\right)$ is free on 1 and $a_{k}=\varphi_{R / x_{k}}^{G}\left(\bar{x}_{k}\right)$, where $\varphi_{R / x_{k}}^{G}$ is the characteristic homomorphism of the admissible pair $\left(R / x_{k}, G, \pi_{k}\right)$. Therefore, the Kronecker duality homomorphism (see eg [5, Proposition 2.25])

$$
d: G_{R}^{*}\left(R / x_{k} \wedge R / x_{k}\right) \longrightarrow \operatorname{Hom}_{G_{*}}^{*}\left(G_{*}^{R}\left(R / x_{k} \wedge R / x_{k}\right), G_{*}\right)
$$

is an isomorphism. To relieve the notation, we identify $G_{*}^{R}\left(R / x_{k} \wedge R / x_{k}\right)$ with $G_{*}^{R}\left(R / x_{k}\right) \otimes G_{*}^{R}\left(R / x_{k}\right)$ via the Künneth isomorphism with respect to $\mu_{G}$ in the following.

To show that $\pi_{k}$ is multiplicative, we need to verify that $d\left(\mu_{G}\left(\pi_{k} \wedge \pi_{k}\right)\right)$ and $d\left(\pi_{k} \mu_{k}\right)$ take the same values on the basis elements $1 \otimes 1,1 \otimes a_{k}, a_{k} \otimes 1$ and $a_{k} \otimes a_{k}$ of $G_{*}^{R}\left(R / x_{k}\right) \otimes G_{*}^{R}\left(R / x_{k}\right)$. By naturality of the characteristic homomorphism, we have that $G_{*}^{R}\left(\pi_{k}\right)\left(a_{k}\right)=\varphi_{G}\left(\pi_{*}\left(\bar{x}_{k}\right)\right) \in G_{*}^{R}(G)$, where $\pi_{*}$ denotes the homomorphism $\pi_{*}: G_{*} \otimes_{F_{*}} I / I^{2}[1] \rightarrow J / J^{2}[1]$ induced by $\pi$. Writing $a_{k}^{\prime}$ for this element and suppressing Künneth isomorphisms from the notation, we compute

$$
\begin{aligned}
d\left(\mu_{G}\left(\pi_{k} \wedge \pi_{k}\right)\right)\left(a_{k} \otimes a_{k}\right) & =\left(\mu_{G}\right)_{*} G_{*}^{R}\left(\mu_{G}\right)\left(G_{*}^{R}\left(\pi_{k}\right) \otimes G_{*}^{R}\left(\pi_{k}\right)\right)\left(a_{k} \otimes a_{k}\right) \\
& =\left(\mu_{G}\right)_{*} G_{*}^{R}\left(\mu_{G}\right)\left(a_{k}^{\prime} \otimes a_{k}^{\prime}\right) \\
& =b_{G}\left(\pi_{*}\left(\bar{x}_{k}\right) \otimes \pi_{*}\left(\bar{x}_{k}\right)\right)=\pi^{*}\left(b_{G}\right)\left(\bar{x}_{k} \otimes \bar{x}_{k}\right) .
\end{aligned}
$$


On the other hand, denoting both the residue classes of $x_{k}$ in $G_{*} \otimes_{R_{*}} / x_{k}\left(x_{k}\right) /\left(x_{k}\right)^{2}[1]$ and in $G_{*} \otimes_{F_{*}} I / I^{2}[1]$ by $\bar{x}_{k}$, we have

$d\left(\pi_{k} \mu_{k}\right)\left(a_{k} \otimes a_{k}\right)=\left(\mu_{G}\right)_{*} G_{*}^{R}\left(\pi_{k} \mu_{k}\right)\left(a_{k} \otimes a_{k}\right)=b_{R / x_{k}}^{G}\left(\bar{x}_{k} \otimes \bar{x}_{k}\right)=b_{F}^{G}\left(\bar{x}_{k} \otimes \bar{x}_{k}\right)$.

For the last equality, we have used that $j_{k}: R / x_{k} \rightarrow F$ is multiplicative. By the hypothesis, we have $\pi^{*}\left(b_{G}\right)=b_{F}^{G}$, which shows that

$$
d\left(\mu_{G}\left(\pi_{k} \wedge \pi_{k}\right)\right)\left(a_{k} \otimes a_{k}\right)=d\left(\pi_{k} \mu_{k}\right)\left(a_{k} \otimes a_{k}\right) .
$$

Similar, but simpler calculations show that $d\left(\mu_{G}\left(\pi_{k} \wedge \pi_{k}\right)\right)$ and $d\left(\pi_{k} \mu_{k}\right)$ agree on the other basis elements $1 \otimes 1,1 \otimes a_{k}$ and $a_{k} \otimes 1$ as well.

In a second step, we prove that $\pi_{k}$ and $\pi_{l}$ commute for $k \neq l$, in the sense that $\mu_{G}\left(\pi_{k} \wedge \pi_{l}\right)=\mu_{G}^{\mathrm{op}}\left(\pi_{k} \wedge \pi_{l}\right)$. The relevant Kronecker duality morphism

$$
d: G_{R}^{*}\left(R / x_{k} \wedge R / x_{l}\right) \longrightarrow \operatorname{Hom}_{G_{*}}^{*}\left(G_{*}^{R}\left(R / x_{k} \wedge R / x_{l}\right), G_{*}\right)
$$

is again an isomorphism. We use the notation and conventions from above and evaluate $d\left(\mu_{G}^{\mathrm{op}}\left(\pi_{k} \wedge \pi_{l}\right)\right)$ and $d\left(\mu_{G}\left(\pi_{k} \wedge \pi_{l}\right)\right)$ on $a_{k} \otimes a_{l}$. We first compute

$$
\begin{aligned}
d\left(\mu_{G}^{\mathrm{op}}\left(\pi_{k} \wedge \pi_{l}\right)\right)\left(a_{k} \otimes a_{l}\right) & =\left(\mu_{G}\right)_{*} G_{*}^{R}\left(\mu_{G}^{\mathrm{op}}\right)\left(G_{*}^{R}\left(\pi_{k}\right) \otimes G_{*}^{R}\left(\pi_{l}\right)\right)\left(a_{k} \otimes a_{l}\right) \\
& =\left(\mu_{G}\right)_{*} G_{*}^{R}\left(\mu_{G}^{\mathrm{op}}\right)\left(a_{k}^{\prime} \otimes a_{l}^{\prime}\right)=\left(\mu_{G}\right)_{*}\left(a_{k}^{\prime} * a_{l}^{\prime}\right),
\end{aligned}
$$

where $*$ denotes the product on $G_{*}^{R}\left(G^{\mathrm{op}}\right)$. Now $\left(\mu_{G}\right)_{*}: G_{*}^{R}\left(G^{\mathrm{op}}\right) \rightarrow G_{*}$ is multiplicative by [5, Corollary 3.3]. Together with $G_{\text {odd }}=0$, this implies that $\left(\mu_{G}\right)_{*}\left(a_{k} * a_{l}\right)=$ $\left(\mu_{G}\right)_{*}\left(a_{k}\right) \cdot\left(\mu_{G}\right)_{*}\left(a_{l}\right)=0$. On the other hand, we have

$$
\begin{aligned}
d\left(\mu_{G}\left(\pi_{k} \wedge \pi_{l}\right)\right)\left(a_{k} \otimes a_{l}\right) & =\left(\mu_{G}\right)_{*} G_{*}^{R}\left(\mu_{G}\right)\left(a_{k}^{\prime} \otimes a_{l}^{\prime}\right) \\
& =\left(\mu_{G}\right)_{*}\left(a_{k}^{\prime} \cdot a_{l}^{\prime}\right) \\
& =b_{G}\left(\bar{\pi}_{*}\left(\bar{x}_{k}\right) \otimes \bar{\pi}_{*}\left(\bar{x}_{l}\right)\right)=\pi^{*}\left(b_{G}\right)\left(\bar{x}_{k} \otimes \bar{x}_{l}\right),
\end{aligned}
$$

where $\cdot$ denotes the product of $G_{*}^{R}(G)$. Since $\pi^{*}\left(b_{G}\right)=G_{*} \otimes b_{F}$ by hypothesis, since $b_{F}$ is diagonal with respect to the basis $\bar{x}_{1}, \bar{x}_{2}, \ldots$ and since $k \neq l$, we have $\pi^{*}\left(b_{G}\right)\left(\bar{x}_{k} \otimes \bar{x}_{l}\right)=0$.

Leaving the analogous, simpler computations on $1 \otimes 1,1 \otimes a_{l}, a_{k} \otimes 1$ again to the reader, we conclude that $d\left(\mu_{G}^{\mathrm{op}}\left(\pi_{k} \wedge \pi_{l}\right)\right)=d\left(\mu_{G}\left(\pi_{k} \wedge \pi_{l}\right)\right)$. Hence $\pi_{k}$ and $\pi_{l}$ commute with each other, which concludes the proof.

By [5, Proposition 2.35], the characteristic bilinear form of a diagonal regular quotient ring is diagonal. We now show that the converse is true as well: 
Proposition 6.4 Let $F=R / I$ be a regular quotient ring and $\left(x_{1}, x_{2}, \ldots\right)$ a regular sequence generating the ideal $I$. Then $F$ is diagonal with respect to the sequence $\left(x_{1}, x_{2}, \ldots\right)$ if and only if $b_{F}$ is diagonal with respect to the basis $\bar{x}_{1}, \bar{x}_{2}, \ldots$ of $I / I^{2}[1]$.

Proof The necessity of the condition was shown in [5], as noted above. For sufficiency, assume that $b_{F}$ is diagonal, and let $\mu_{k}$ be a product on $R / x_{k}$ such that the canonical map $j_{k}: R / x_{k} \rightarrow F$ is multiplicative, for all $k$. The proof of Proposition 6.3 above shows that $j_{k}$ and $j_{l}$ commute if $k \neq l$, since $b_{F}$ is diagonal with respect to the $\bar{x}_{i}$. From [9, Proposition 4.8], we deduce that the product on $F$ is the smash ring product of the $\mu_{k}$.

Lemma 6.5 Let $(F, G, \pi)$ be an admissible pair satisfying the conditions of Theorem 6.2. Assume that $G_{*} \otimes b_{F}=\pi^{*}\left(b_{G}\right)$.

(i) There exist products $\bar{\mu}$ on $F$ and $\bar{v}$ on $G$ such that $\pi: \bar{F} \rightarrow \bar{G}$ is multiplicative.

(ii) For any $d \in \operatorname{Der}_{R}^{*}(G)$ there exists $\delta \in \operatorname{Der}_{R}^{*}(F)$ such that $d \pi=\pi \delta$.

Proof (i) Let $\beta \in \operatorname{Bil}\left(I / I^{2}[1]\right)$ be defined by $\beta\left(\bar{x}_{i} \otimes \bar{x}_{j}\right)=0$ for $i \geqslant j, \beta\left(\bar{x}_{i} \otimes \bar{x}_{j}\right)=$ $b_{F}\left(\bar{x}_{i} \otimes \bar{x}_{j}\right)$ for $i<j$ and let $\widetilde{F}=\beta F$. By Corollary 5.3, the characteristic bilinear form $b_{\widetilde{F}}$ of $\tilde{F}$ is given by $b_{\widetilde{F}}=b_{F}-\left(\beta+\beta^{t}\right)$ and is therefore diagonal with respect to the $\bar{x}_{i}$, as $b_{F}$ is symmetric by Corollary 5.4.

Since $(F, G, \pi)$ is smooth, the homomorphism

$$
\pi^{*}: \operatorname{Bil}\left(J / J^{2}[1]\right) \rightarrow \operatorname{Bil}\left(G_{*} \otimes F_{*} I / I^{2}[1]\right)
$$

is surjective. Choose $\gamma \in \operatorname{Bil}\left(J / J^{2}[1]\right)$ with $\pi^{*}(\gamma)=G_{*} \otimes \beta$ and set $\bar{G}=\gamma G$. By hypothesis and by Corollary 5.3, it follows that $G_{*} \otimes b_{\widetilde{F}}=\pi^{*}\left(b_{\bar{G}}\right)$.

Let $\pi_{k}=\pi j_{k}: R / x_{k} \rightarrow \bar{G}$, with $j_{k}$ the canonical map. The proof of Proposition 6.3 implies that $\pi_{k}$ and $\pi_{l}$ commute for $k \neq l$. Choose a product $\mu_{k}$ on $R / x_{k}$ such that $\pi_{k}$ is multiplicative, for each $k$, and let $\bar{F}$ be the induced smash ring spectrum. By [9, Proposition 4.8], $\pi: \bar{F} \rightarrow \bar{G}$ is then multiplicative.

(ii) Suppose first that $\pi$ is multiplicative. Then we have the commutative diagram

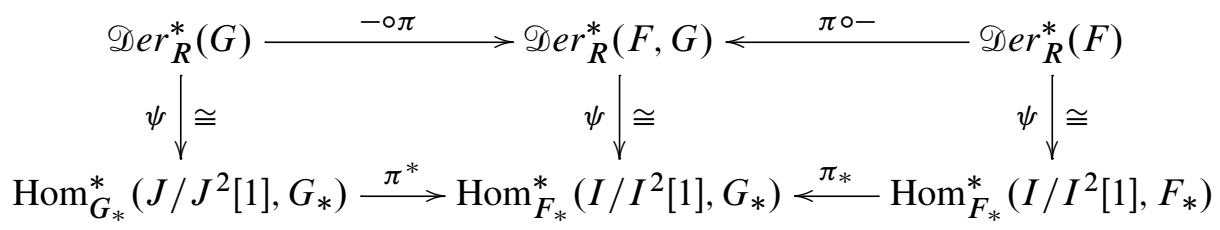

where $\psi$ is as in (2-4). The right bottom morphism $\pi_{*}$ is surjective, which implies the statement in this particular case. 
In the general case, (i) implies that there exist products $\bar{\mu}$ on $F$ and $\bar{v}$ on $G$ such that $\pi: \bar{F} \rightarrow \bar{G}$ is multiplicative. By [5, Lemma 4.6], $d$ is a derivation for any product on $G$, in particular $d \in \operatorname{Der}_{R}^{*}(\bar{G})$. By what we have shown above, there exists $\delta \in \operatorname{Der}_{R}^{*}(\bar{F})$ such that $d \pi=\pi \delta$. By [5, Lemma 4.6] again, we deduce that $\delta \in \operatorname{Der}_{R}^{*}(F)$, which proves (ii).

The following two statements are generalizations of Lemma 5.8 and Proposition 5.2, respectively, for the case where the map of the admissible pair is not necessarily the identity.

Lemma 6.6 For an admissible pair $(F, G, \pi)$ satisfying the conditions of Theorem 6.2 and $\gamma \in \operatorname{Bil}\left(J / J^{2}[1]\right)$, we have $b_{F}^{\gamma G}=b_{F}^{G}-\pi^{*}\left(\gamma^{t}\right)$.

Proof Let $\varphi=\varphi_{F}^{G}$ be the characteristic homomorphism of the admissible pair $(F, G, \pi)$. In a first step, we show that for $x, y \in I$, we have

$$
m_{F}^{\gamma G}(\varphi(\bar{x}) \otimes \varphi(\bar{y}))=m_{F}^{G}(\varphi(\bar{x}) \otimes \varphi(\bar{y}))-\pi^{*}(\gamma)(\bar{x} \otimes \bar{y}) \cdot 1 .
$$

Let $\mu$ be the product on $F$ and $\nu$ the one on $G$, and let us write $a \cdot b$ for $m_{F}^{G}(a \otimes b) \in$ $G_{*}^{R}(F)$, where $a, b \in G_{*}^{R}(F)$. Clearly, it suffices to prove (6-1) for the case where $\gamma$ is of the form $\gamma=\alpha \otimes \alpha^{\prime}$, with $\alpha, \alpha^{\prime} \in D\left(J / J^{2}[1]\right)$. Let $d, d^{\prime} \in \operatorname{Der}_{R}^{*}(G)$ correspond to $\alpha, \alpha^{\prime}$, respectively, under the isomorphism $\psi$ : $\operatorname{Der}_{R}^{*}(G) \rightarrow D\left(J / J^{2}[1]\right)$. We have $\gamma v=v+v\left(d \wedge d^{\prime}\right)$. Recall that for $x \in I$, we denote both the residue classes of $x \in I$ in $I / I^{2}[1]$ and in $G_{*} \otimes_{F_{*}} I / I^{2}[1]$ by $\bar{x}$. Exactly as in the proof of Lemma 5.8 we identify $m_{F}^{\gamma G}(\varphi(\bar{x}) \otimes \varphi(\bar{y}))$ for $x, y \in I$ as

$$
\varphi(\bar{x}) \cdot \varphi(\bar{y})-(\mu \wedge \nu)_{*}(1 \wedge \tau \wedge 1)_{*} \zeta\left((d \wedge 1)_{*}(\varphi(\bar{x})) \otimes\left(d^{\prime} \wedge 1\right)_{*}(\varphi(\bar{y}))\right) .
$$

To determine $(d \wedge 1)_{*}(\varphi(\bar{x}))$, we proceed as follows. By Lemma 6.5(ii), there exists $\delta \in \operatorname{Der}_{R}^{*}(F)$ such that $\pi \delta=d \pi$. By commutativity of the diagram

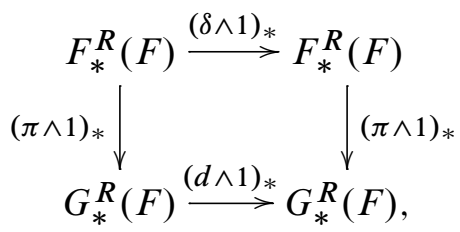

and using similar arguments as in the proof of Lemma 5.8, we deduce

$$
\begin{aligned}
(d \wedge 1)_{*}(\varphi(\bar{x})) & =(d \wedge 1)_{*}\left(\varphi_{F}^{G}(\bar{x})\right)=(\pi \wedge 1)_{*}(\delta \wedge 1)_{*}\left(\varphi_{F}(\bar{x})\right) \\
& =(\pi \wedge 1)_{*} \tau_{*}(1 \wedge \delta) \tau_{*}\left(\varphi_{F}(\bar{x})\right)=-(\pi \wedge 1)_{*}(\psi(\delta)(\bar{x}) \cdot 1) \\
& =-\pi_{*}(\psi(\delta)(\bar{x})) \cdot 1=-\pi^{*}(\psi(d))(\bar{x}) \cdot 1=-\pi^{*}(\alpha)(\bar{x}) \cdot 1 .
\end{aligned}
$$


Similarly, we obtain $\left(d^{\prime} \wedge 1\right)_{*}(\varphi(\bar{y}))=-\pi^{*}\left(\alpha^{\prime}\right)(\bar{y}) \cdot 1$. It follows that

$$
m_{F}^{\gamma G}(\varphi(\bar{x}) \otimes \varphi(\bar{y}))=\varphi(\bar{x}) \cdot \varphi(\bar{y})-\pi^{*}(\alpha)(\bar{x}) \pi^{*}\left(\alpha^{\prime}\right)(\bar{y}) \cdot 1,
$$

which is (6-1) for $\gamma=\alpha \otimes \alpha^{\prime}$.

We now proceed to the proof of the lemma itself. Again, we assume $\gamma=\alpha \otimes \alpha^{\prime}$, with $\alpha, \alpha^{\prime} \in D\left(J / J^{2}[1]\right)$, and let $d=\psi(\alpha), d^{\prime}=\psi\left(\alpha^{\prime}\right) \in \mathscr{D e r}{ }_{R}^{*}(G)$. Using (6-1), we start the computation of $b_{F}^{\gamma G}(\bar{x} \otimes \bar{y})$ for $x, y \in I$ as in the proof of Proposition 5.2 and find

$$
b_{F}^{\gamma G}(\bar{x} \otimes \bar{y})=b_{F}^{G}(\bar{x} \otimes \bar{y})-\pi^{*}(\gamma)(\bar{x} \otimes \bar{y})+\left(\nu\left(d \wedge d^{\prime}\right)(1 \wedge \pi)\right)_{*}(\varphi(\bar{x}) \cdot \varphi(\bar{y})) .
$$

We now identify the last summand of the sum on the right hand side. Since $d^{\prime} \in$ $\operatorname{Der}_{R}^{*}(G)$ is a derivation, the homomorphism

$$
\left(1 \wedge d^{\prime}\right)_{*}=G_{*}^{R}\left(d^{\prime}\right): G_{*}^{R}(G) \rightarrow G_{*}^{R}(G)
$$

is a derivation, too [5, Lemma 4.3]. Using [5, Lemma 4.11], and writing $\cdot$ for $m_{G}^{G}$ (as well as for $m_{F}^{G}$ ), we compute

$$
\begin{aligned}
\left(v\left(d \wedge d^{\prime}\right)(1 \wedge\right. & \pi))_{*}(\varphi(\bar{x}) \cdot \varphi(\bar{y})) \\
& =v_{*}(d \wedge 1)_{*}\left(1 \wedge d^{\prime}\right)_{*}\left(\varphi_{G}(\bar{x}) \cdot \varphi_{G}(\bar{y})\right) \\
& =v_{*}(d \wedge 1)_{*}\left(G_{*}^{R}\left(d^{\prime}\right)\left(\varphi_{G}(\bar{x})\right) \cdot \varphi_{G}(\bar{y})-\varphi_{G}(\bar{x}) \cdot G_{*}^{R}\left(d^{\prime}\right)\left(\varphi_{G}(\bar{y})\right)\right) \\
& =v_{*}(d \wedge 1)_{*}\left(\pi^{*}\left(\alpha^{\prime}\right)(\bar{x}) \varphi_{G}(\bar{y})-\varphi_{G}(\bar{x}) \pi^{*}\left(\alpha^{\prime}\right)(\bar{y})\right) .
\end{aligned}
$$

In the proof of (6-1) above, we showed that $-(d \wedge 1)_{*}(\varphi(\bar{x}))=-\pi^{*}(\alpha)(\bar{x}) \cdot 1$. Using the analogous expression for $(d \wedge 1)_{*}(\varphi(\bar{y}))$, we find that

$$
b_{F}^{\beta G}(\bar{x} \otimes \bar{y})=b_{F}^{G}(\bar{x} \otimes \bar{y})-\pi^{*}\left(\alpha^{\prime}\right)(\bar{x}) \pi^{*}(\alpha)(\bar{y}) .
$$

This finishes the proof of the lemma.

Proof of Theorem 6.2 If $\pi$ is multiplicative, then $(F, G, \pi)$ is a multiplicative admissible pair and the statement follows from [5, Proposition 2.20].

Conversely, let us assume that $b_{F}^{G}=\pi^{*}\left(b_{G}\right)=G_{*} \otimes b_{F}$. Let $\mu$ be the product on $F$ and $v$ the one on $G$. Let $\left(x_{1}, x_{2}, \ldots\right)$ be a regular sequence generating the ideal $I$. Let $\bar{\mu}$ be a product on $F$ which is diagonal with respect to $\left(x_{1}, x_{2}, \ldots\right)$ (see eg [5, Corollary 2.10]) and let $\beta \in \operatorname{Bil}\left(I / I^{2}[1]\right)$ be such that $\bar{F}=\beta F$. Write $\beta$ as a sum $\sum_{i} \varepsilon_{i} \otimes \varepsilon_{i}^{\prime}$ with $\varepsilon_{i}, \varepsilon_{i}^{\prime} \in D_{F_{*}}\left(I / I^{2}[1]\right)$. Because $(F, G, \pi)$ is smooth, the composition

$$
\pi^{*}: D_{G_{*}}\left(J / J^{2}[1]\right) \rightarrow D_{G_{*}}\left(G_{*} \otimes I / I^{2}[1]\right) \cong G_{*} \otimes F_{*} D_{F_{*}}\left(I / I^{2}[1]\right)
$$

is surjective. Choose $\alpha_{i}, \alpha_{i}^{\prime} \in D_{G_{*}}\left(J / J^{2}[1]\right)$ such that $\pi^{*}\left(\alpha_{i}\right)=\varepsilon_{i}$ and $\pi^{*}\left(\alpha_{i}^{\prime}\right)=\varepsilon_{i}^{\prime}$ and define $\gamma=\sum \alpha_{i} \otimes \alpha_{i}^{\prime}$. Observe that $\pi^{*}(\gamma)=G_{*} \otimes \beta$. 
Now set $\bar{G}=\gamma G$. Using Proposition 5.1 and Lemma 6.6, we compute

$$
b_{\bar{F}}^{\bar{G}}=b_{\beta F}^{\gamma G}=b_{F}^{G}-\pi^{*}(\gamma)^{t}-G_{*} \otimes \beta=\pi^{*}\left(b_{G}-\gamma^{t}-\gamma\right)=\pi^{*}\left(b_{\bar{G}}\right) .
$$

Similarly, we find

and so

$$
b_{\bar{F}}^{\bar{G}}=G_{*} \otimes b_{\bar{F}},
$$

$$
G_{*} \otimes b_{\bar{F}}=b_{\bar{F}}^{\bar{G}}=\pi^{*}\left(b_{\bar{G}}\right) .
$$

Since $\bar{F}$ is diagonal, this implies by Proposition 6.3 that $\pi: \bar{F} \rightarrow \bar{G}$ is multiplicative. Let $d_{i}, d_{i}^{\prime} \in \operatorname{Der}_{R}^{*}(G)$ be the derivations corresponding to $\alpha_{i}, \alpha_{i}^{\prime}$ under the isomorphism $\psi: \operatorname{Der}_{R}^{*}(G) \cong D_{G_{*}}\left(J / J^{2}[1]\right)$, and $\delta_{i}, \delta_{i}^{\prime} \in \operatorname{Der}_{R}^{*}(F)$ corresponding under $\psi: \operatorname{Der}_{R}^{*}(F) \cong D_{F_{*}}\left(I / I^{2}[1]\right)$ to $\varepsilon_{i}, \varepsilon_{i}^{\prime}$. By naturality of $\psi$, we have $d_{i} \pi=\pi \delta_{i}$ and $d_{i}^{\prime} \pi=\pi \delta_{i}^{\prime}$. From the definition of the canonical action of the group of bilinear forms on the set of products, we have that

$$
\begin{aligned}
\gamma \bar{v} \circ(\pi \wedge \pi) & =\bar{v} \circ \prod_{i}\left(1+d_{i} \wedge d_{i}^{\prime}\right)(\pi \wedge \pi) \\
& =\bar{v} \circ(\pi \wedge \pi) \circ \prod_{i}\left(1+\delta_{i} \wedge \delta_{i}^{\prime}\right)=\pi \bar{\mu} \circ \prod_{i}\left(1+\delta_{i} \wedge \delta_{i}^{\prime}\right)=\pi \circ \beta \bar{\mu} .
\end{aligned}
$$

Therefore $\pi: F=-\beta \bar{F} \rightarrow-\gamma \bar{G}=G$ is multiplicative. This completes the proof of the theorem.

\section{Classification of products up to equivalence}

In this section, we classify the products on regular quotients up to equivalence. Moreover, we study commutative products and consider the question of diagonalizability of products on regular quotients.

Let $F=R / I$ be a regular quotient ring with product $\mu$. If $\bar{\mu}$ is a second product on $F$, we write $\bar{F}$ for $F$, endowed with $\bar{\mu}$, as before. If $\beta \in \operatorname{Bil}\left(I / I^{2}[1]\right)$ is such that $\bar{\mu}=\beta \mu$, we alternatively write $\bar{F}=\beta F$.

Recall the following definition:

Definition 7.1 Two products $\mu$ and $\bar{\mu}$ on $F$ are equivalent (denoted $\mu \sim \bar{\mu}$ ) if there is a multiplicative isomorphism $f: F \rightarrow \bar{F}$ in $\mathscr{D}_{R}$. Such a map $f$ is called a multiplicative equivalence.

Together with Theorem 4.1, the following result gives a classification for products up to equivalence: 
Theorem 7.2 Let $F=R / I$ be a regular quotient ring and $\beta \in \operatorname{Bil}\left(I / I^{2}[1]\right)$ a bilinear form. Then $F$ and $\beta F$ are equivalent if and only if $\beta$ is alternating. In this case, there is a canonical multiplicative equivalence $F \rightarrow \beta F$.

Let $F=R / I$ be a regular quotient ring. Consider the map

$$
\theta:\left(\operatorname{Der}_{R}^{*}(F) \times \operatorname{Der}_{R}^{*}(F)\right)^{0} \rightarrow F_{R}^{0}(F)
$$

defined by $\theta\left(d, d^{\prime}\right)=1+d d^{\prime}$. Since $F_{R}^{*}(F) \cong \widehat{\Lambda}\left(D e r_{R}^{*}(F, F)\right.$ ) (by (2-5)), the image of $\theta$ is contained in the center of the monoid $F_{R}^{*}(F)$, the product on $F_{R}^{*}(F)$ being the composition. Clearly, $\theta$ is bilinear. Since $F_{R}^{*}(F)$ is complete with respect to the profinite topology, $\theta$ induces (see (3-1))

$$
\Theta: \operatorname{Bil}\left(I / I^{2}[1]\right) \cong\left(\operatorname{Der}_{R}^{*}(F) \hat{\otimes} \operatorname{Der}_{R}^{*}(F)\right)^{0} \longrightarrow F_{R}^{0}(F) .
$$

The next lemma is a crucial step in the proof of Theorem 7.2.

Lemma 7.3 Let $F=R / I$ be a regular quotient ring and $\beta \in \operatorname{Alt}\left(I / I^{2}[1]\right)$. Then $\Theta(\beta)$ is a multiplicative equivalence $\Theta(\beta): F \rightarrow \beta F$.

Proof It suffices to prove the lemma for bilinear forms $\beta$ of the form $\beta=\alpha \otimes \alpha^{\prime}-\alpha^{\prime} \otimes \alpha$ with $\alpha, \alpha^{\prime} \in D\left(I / I^{2}[1]\right)$, because an arbitrary alternating bilinear form can be written as a sum of such elements. Let $d, d^{\prime} \in \operatorname{Der}_{R}^{*}(F)$ correspond to $\alpha, \alpha^{\prime}$ under the isomorphism $\psi$ : $\operatorname{Der}_{R}^{*}(F) \cong D\left(I / I^{2}[1]\right)$ (2-4). Denoting by $\mu$ the product on $F$, we then have $\beta \mu=\mu\left(1+d \wedge d^{\prime}\right)\left(1-d^{\prime} \wedge d\right)$. In order to simplify the notation, we write $\bar{\mu}$ for $\beta \mu$.

Since derivations anticommute, the map $f=1+d d^{\prime}$ is an equivalence, with inverse $1-d d^{\prime}$. We have to show that $f: F \rightarrow \bar{F}$ is multiplicative, that is, $f \mu=\bar{\mu}(f \wedge f)$. For this, we first compute

$$
\begin{aligned}
f \mu=\left(1+d d^{\prime}\right) \mu & =\mu\left(1+(d \wedge 1+1 \wedge d)\left(d^{\prime} \wedge 1+1 \wedge d^{\prime}\right)\right) \\
& =\mu\left(1+d d^{\prime} \wedge 1+d \wedge d^{\prime}-d^{\prime} \wedge d+1 \wedge d d^{\prime}\right) .
\end{aligned}
$$

On the other hand, we find

$$
\begin{aligned}
\bar{\mu}(f \wedge f) & =\mu\left(1+d \wedge d^{\prime}\right)\left(1-d^{\prime} \wedge d\right)\left(1+d d^{\prime} \wedge 1+1 \wedge d d^{\prime}+d d^{\prime} \wedge d d^{\prime}\right) \\
& =\mu\left(1+d d^{\prime} \wedge 1+1 \wedge d d^{\prime}+d d^{\prime} \wedge d d^{\prime}-d^{\prime} \wedge d+d \wedge d^{\prime}+d d^{\prime} \wedge d^{\prime} d\right) .
\end{aligned}
$$

Since $d d^{\prime}=-d^{\prime} d$, the lemma is proven.

Proof of Theorem 7.2 We fix a regular sequence $\left(x_{1}, x_{2}, \ldots\right)$ generating $I$. The residue classes $\bar{x}_{1}, \bar{x}_{2}, \ldots$ form a basis of $V=I / I^{2}[1]$, and we denote by $\bar{x}_{1}^{\vee}, \bar{x}_{2}^{\vee}, \ldots$ 
the elements dual to the $\bar{x}_{i}$. The Bockstein operations $Q_{i}$ are defined as $Q_{i}=\psi^{-1}\left(\bar{x}_{i}^{\vee}\right)$, where $\psi$ is the isomorphism $\psi: \operatorname{Der}_{R}^{*}(F) \rightarrow D(V)$.

Assume first that $\beta$ is alternating. Then it can be written as $\beta=\sum v_{i j} \bar{x}_{i}^{\vee} \otimes \bar{x}_{j}^{\vee}$ with $v_{i i}=0$ and $v_{i j}=-v_{j i}$ for $i \neq j$. Thus, $\beta \mu$ can be expressed as (see Lemma 4.2)

$$
\beta \mu=\mu \prod_{i<j}\left(\left(1+v_{i j} Q_{i} \wedge Q_{j}\right)\left(1-v_{i j} Q_{j} \wedge Q_{i}\right)\right) .
$$

If the product in (7-1) is finite, the map $f=\prod_{i<j}\left(1+v_{i j} Q_{i} Q_{j}\right)$ is a multiplicative homotopy equivalence $f: F \rightarrow \beta F$ by Lemma 7.3. If the product in (7-1) is infinite, the Cauchy sequence of multiplicative equivalences $\left(\prod_{i<j, i+j \leqslant k}\left(1+v_{i j} Q_{i} Q_{j}\right)\right)_{k}$ converges to one from $F$ to $\beta F$.

Suppose now that $F$ and $\bar{F}=\beta F$ are equivalent via a multiplicative equivalence $\pi: F \rightarrow \bar{F}$. Since both $\pi_{*}: F_{*} \rightarrow \bar{F}_{*}$ and the homomorphism $\bar{\pi}_{*}: \bar{F}_{*} \otimes I / I^{2}[1] \cong$ $I / I^{2}[1] \rightarrow I / I^{2}[1]$ induced by $\pi_{*}$ are (equivalent to) the identities, naturality of the characteristic bilinear form and the commutative diagram

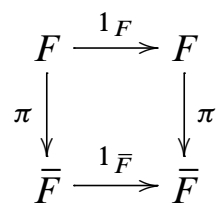

show that $b_{F}=b_{\bar{F}}$. Corollary 5.3 implies that $b_{\bar{F}}=b_{F}-\left(\beta+\beta^{t}\right)$. It follows that $\beta$ is antisymmetric. Hence it remains to check that $\beta\left(\bar{x}_{i} \otimes \bar{x}_{i}\right)=0$ for all $i$ in order to prove that $\beta$ is alternating.

Choose a product on $R / x_{i}$ such that the natural map $j_{i}: R / x_{i} \rightarrow F$ is multiplicative. Then both $\left(R / x_{i}, F, j_{i}\right)$ and $\left(R / x_{i}, \bar{F}, \pi j_{i}\right)$ are multiplicative admissible pairs, and [5, Proposition 2.21] implies that

$$
b_{\left(R / x_{i}\right)^{\mathrm{op}}}^{F}=0=b_{\left(R / x_{i}\right)^{\mathrm{op}}}^{\bar{F}} .
$$

Since $\left(x_{i}\right) \subseteq I$ is smooth, Lemma 6.6 applies. On setting $b_{i}=b_{R / x_{i}}$ and recalling that $\left(R / x_{i}\right)^{\mathrm{op}}=b_{i} R / x_{i}$ (Corollary 5.5), we obtain

$$
\begin{aligned}
0=b_{\left(R / x_{i}\right)^{\text {op }}}^{\bar{F}}=b_{b_{i} R / x_{i}}^{\beta F}=b_{R / x_{i}}^{\beta F}-F_{*} \otimes b_{i} & =b_{R / x_{i}}^{F}-F_{*} \otimes b_{i}-j_{i}^{*}\left(\beta^{t}\right) \\
& =b_{b_{i} R / x_{i}}^{F}+j_{i}^{*}(\beta) \\
& =b_{\left(R / x_{i}\right)^{\text {op }}}^{F}+j_{i}^{*}(\beta)=j_{i}^{*}(\beta) .
\end{aligned}
$$

Therefore $0=j_{i}^{*}(\beta)\left(\bar{x}_{i} \otimes \bar{x}_{i}\right)=\beta\left(\bar{x}_{i} \otimes \bar{x}_{i}\right)$, where $\bar{x}_{i}$ again stands for the residue class of $x_{i}$ in either $\left(x_{i}\right) /\left(x_{i}\right)^{2}[1]$ or $I / I^{2}[1]$. Thus $\beta$ is alternating, and the theorem is proven. 
Remark 7.4 Theorem 7.2 states that $\operatorname{Alt}\left(I / I^{2}[1]\right)$ acts freely and transitively on the equivalence class of any product on $F$. Therefore, the (additive) group of quadratic forms $\mathrm{QF}\left(I / I^{2}[1]\right) \cong \operatorname{Bil}\left(I / I^{2}[1]\right) / \operatorname{Alt}\left(I / I^{2}[1]\right)$ acts freely and transitively on the set of equivalence classes of products on $F$.

Corollary 7.5 Let $\mu$ and $\bar{\mu}$ be two products on a regular quotient $F$.

(i) If $F$ and $\bar{F}$ are equivalent then $b_{F}=b_{\bar{F}}$.

(ii) If $F_{*}$ is 2-torsion-free, then $F$ and $\bar{F}$ are (canonically) equivalent if and only if $b_{F}=b_{\bar{F}}$ if and only if $q_{F}=q_{\bar{F}}$.

Proof (i) This has been shown in the proof of Theorem 7.2.

(ii) Suppose that $b_{F}=b_{\bar{F}}$. Let $\beta \in \operatorname{Bil}\left(I / I^{2}[1]\right)$ be the bilinear form which satisfies $\beta F=\bar{F}$ (Theorem 4.1). As in the proof of Theorem 7.2, we deduce that $\beta^{t}=-\beta$. As $F_{*}$ is 2 -torsion-free, this means that $\beta$ is alternating. Now Theorem 7.2 implies that $F$ and $\beta F=\bar{F}$ are equivalent. The last implication is clear.

Remark 7.6 If $F_{*}$ has 2-torsion, there may exist nonequivalent products $\mu, \bar{\mu} \in$ $\operatorname{Prod}_{R}(F)$ with $b_{F}=b_{\bar{F}}$; see for instance Proposition 8.7.

Remark 7.7 Let $F=R / I$ be a regular quotient ring. We may interpret the characteristic bilinear form as a map $b: \operatorname{Prod}_{R}(F) \rightarrow \operatorname{Bil}\left(I / I^{2}[1]\right)$. By Corollary 5.4, the image of $b$ is contained in $\operatorname{Sym}\left(I / I^{2}[1]\right) \subseteq \operatorname{Bil}\left(I / I^{2}[1]\right)$, and by Corollary 7.5, $b$ factors through the set of equivalence classes of products on $F$ :

$$
\bar{b}: \operatorname{Prod}_{R}(F) / \sim \operatorname{Sym}\left(I / I^{2}[1]\right) .
$$

From Corollary 7.5 we deduce that $\bar{b}$ is injective if $F_{*}$ is $2-$ torsion-free. Moreover, we easily check that $\bar{b}$ is surjective if $2 \in F_{*}$ is invertible.

We now turn to a discussion of commutative products on a regular quotient $F$. We prove that if $2 \in F_{*}$ is invertible, there are many commutative products in general (Proposition 7.8), which, however, are all equivalent to each other (Corollary 7.10).

Strickland proves [9, Theorem 2.6] that $F_{*}$ is strongly realizable [9, Definition 2.1] if $2 \in F_{*}$ is invertible. In particular, this shows that $F$ admits a commutative product. Because $F_{*}$ is a quotient of $R_{*}$, any commutative product on $F$ which is equivalent to a strong realization is itself a strong realization. As a consequence, any commutative product turns $F$ into a strong realization of $F_{*}$. 
Proposition 7.8 Let $F=R / I$ be a regular quotient of $R$.

(i) Suppose that $F$ admits a commutative product. Then $\operatorname{Asym}\left(I / I^{2}[1]\right)$ acts freely and transitively on the set of all commutative products.

(ii) If 2 is invertible in $F_{*}$, there exists a commutative product on $F$.

Proof (i) Endow $F$ with a commutative product. For a bilinear form $\beta \in \operatorname{Bil}\left(I / I^{2}[1]\right)$, Corollaries 5.3 and 5.5 imply that $\beta F$ is commutative if and only if $\beta \in \operatorname{Asym}\left(I / I^{2}[1]\right)$. Now the statement follows from Theorem 4.1.

(ii) Let $\mu$ be an arbitrary product on $F$ (see eg [5, Corollary 2.10]) and let $\beta=\frac{1}{2} b_{F}$. Then Corollary 5.3 implies that

$$
b_{\beta F}=b_{F}-\left(\frac{1}{2} b_{F}+\frac{1}{2} b_{F}^{t}\right)=0,
$$

since $b_{F}$ is symmetric. Therefore $\beta F$ is commutative, by Corollary 5.5.

Remark 7.9 Proposition 7.8 is a generalization of [9, Corollary 3.12], which treats the case $F=R / x$. Note that in this situation, $\operatorname{Asym}\left(I / I^{2}[1]\right)$ is the group of 2-torsion elements in $F_{2|x|+2}$.

Using Theorem 7.2, we deduce the following corollary.

Corollary 7.10 Let $F=R / I$ be a regular quotient of $R$.

(i) If $F$ admits a commutative product, then the group $\operatorname{Asym}\left(I / I^{2}[1]\right) / \operatorname{Alt}\left(I / I^{2}[1]\right)$ acts freely and transitively on the set of equivalence classes of commutative products on $F$.

(ii) If $F_{*}$ has no 2-torsion, then there exists at most one commutative product up to canonical equivalence.

(iii) If 2 is invertible in $F_{*}$, there exists a unique commutative product up to canonical equivalence.

Remark 7.11 If $F_{*}$ has 2-torsion, there may not exist any commutative product on $F$. This is well-known; see eg Proposition 8.7.

For regular quotients whose coefficient ring is 2-torsion, we have the following result.

Proposition 7.12 Let $F=R / I$ be a regular quotient such that $2 \cdot F_{*}=0$. Then there exists a commutative product on $F$ if and only if $F$ admits a product whose characteristic bilinear form is alternating. If this holds, then $b\left(\operatorname{Prod}_{R}(F)\right)=\operatorname{Alt}\left(I / I^{2}[1]\right)$, where $b: \operatorname{Prod}_{R}(F) \rightarrow \operatorname{Sym}\left(I / I^{2}[1]\right)$ is the map from Remark 7.7. 
Proof Assume $F$ is endowed with a commutative product. For any $\beta \in \operatorname{Bil}\left(I / I^{2}[1]\right)$, Corollary 5.3 implies that $b_{\beta F}=b_{F}+\beta+\beta^{t}$. Hence for any $\bar{x} \in I / I^{2}[1]$ we have $b_{\beta F}(\bar{x} \otimes \bar{x})=b_{F}(\bar{x} \otimes \bar{x})+2 \beta(\bar{x} \otimes \bar{x})=0$ since $b_{F}=0$. As a consequence $b_{\beta F} \in \operatorname{Alt}\left(I / I^{2}[1]\right)$ and thus $b\left(\operatorname{Prod}_{R}(F)\right) \subseteq \operatorname{Alt}\left(I / I^{2}[1]\right)$.

Conversely, let $F$ be endowed with a product such that $b_{F} \in \operatorname{Alt}\left(I / I^{2}[1]\right)$. Choose a regular sequence $\left(x_{1}, x_{2}, \ldots\right)$ generating the ideal $I$. Define $\beta \in \operatorname{Bil}\left(I / I^{2}[1]\right)$ by $\beta\left(\bar{x}_{i} \otimes \bar{x}_{j}\right)=0$ for $i \leqslant j$ and $\beta\left(\bar{x}_{i} \otimes \bar{x}_{j}\right)=b_{F}\left(\bar{x}_{i} \otimes \bar{x}_{j}\right)$ for $i>j$. Then $b_{\beta F}=b_{F}+\beta+\beta^{t}$ is diagonal with respect to the basis consisting of $\bar{x}_{1}, \bar{x}_{2}, \ldots$. Since $b_{F}$ is alternating, this implies that $b_{\beta F}\left(\bar{x}_{i} \otimes \bar{x}_{i}\right)=b_{F}\left(\bar{x}_{i} \otimes \bar{x}_{i}\right)=0$ and hence that $b_{\beta F}=0$. From Corollary 5.5, it follows that $\beta F$ is commutative.

For the remaining statement, let $F$ be endowed with a commutative product and let $\beta \in \operatorname{Alt}\left(I / I^{2}[1]\right)$ be any alternating bilinear form. With the notation from above, we define $\gamma \in \operatorname{Bil}\left(I / I^{2}[1]\right)$ by $\gamma\left(\bar{x}_{i} \otimes \bar{x}_{j}\right)=0$ for $i \leqslant j$ and $\gamma\left(\bar{x}_{i} \otimes \bar{x}_{j}\right)=\beta\left(\bar{x}_{i} \otimes \bar{x}_{j}\right)$ for $i>j$. Then the characteristic bilinear form of $\gamma F$ satisfies $b_{\gamma F}=b_{F}+\gamma+\gamma^{t}=\beta$, and the proof is complete.

We close this section with a discussion of diagonalizability of products. Recall [5, Definition 2.9] that a regular quotient ring $F$ is diagonalizable if it is equivalent to a diagonal regular quotient ring.

Recall that the maximal ideal of a regular local ring of dimension $n<\infty$ is always generated by a regular sequence of length $n$ [8, Chapter IV].

Proposition 7.13 Assume that $R_{*}$ is a regular local ring of dimension $n$ with maximal ideal $I$ whose residue field $R_{*} / I$ is of characteristic $p \geqslant 0$. Let $F=R / I$.

(i) If $p$ is zero or an odd prime, then $F$ is diagonalizable.

(ii) If $p=2$, then:

(a) If $b_{F} \notin \operatorname{Alt}\left(I / I^{2}[1]\right), F$ is diagonalizable.

(b) If $b_{F} \in \operatorname{Alt}\left(I / I^{2}[1]\right)$ and $b_{F} \neq 0, F$ is not diagonalizable.

(c) If $b_{F}=0, F$ is diagonalizable.

Proof Suppose first that (i) $p$ is zero or odd or that (ii) $p=2$ and $b_{F} \notin \operatorname{Alt}\left(I / I^{2}[1]\right)$. Then [3, Chapter IX, Section 6, Theorem 1] implies that there exists a basis $\mathscr{B}$ consisting of elements $b_{1}, \ldots, b_{n}$ of $I / I^{2}[1]$ such that the matrix of $b_{F}$ with respect to $\mathscr{B}$ is diagonal. By [8, Chapter IV, Proposition 22], there exists a regular sequence $\left(y_{1}, \ldots, y_{n}\right)$ generating $I$ such that $b_{i}=\bar{y}_{i} \in I / I^{2}[1]$ for all $i$. We then conclude with Proposition 6.4. 
Now suppose that $p=2$ and $0 \neq b_{F} \in \operatorname{Alt}\left(I / I^{2}[1]\right)$. For any basis $\mathscr{B}$ consisting of elements $b_{1}, \ldots, b_{n}$ of $I / I^{2}[1]$, we have $b_{F}\left(b_{i} \otimes b_{i}\right)=0$ for all $i$. Therefore, $b_{F}$ is not diagonalizable, since $b_{F} \neq 0$. Hence, by Proposition 6.4 again, $F$ is not diagonalizable.

For the remaining case, $p=2$ and $b_{F}=0$, the statement follows from Proposition 6.4. Alternatively, we may observe that $F$ is commutative (Corollary 5.5) and therefore diagonalizable [5, Corollary 2.12].

\section{Examples}

In this section, we present some applications of our results.

We first collect some facts concerning complex cobordism. Let $M U$ be the commutative $\mathbb{S}$-algebra associated to complex cobordism (see [4]). Recall that there is an isomorphism

$$
M U_{*} \cong \mathbb{Z}\left[x_{1}, x_{2}, \ldots\right],\left|x_{i}\right|=2 i .
$$

Fix a prime number $p$ and recall from [9] that $w_{k} \in M U_{2}\left(p^{k}-1\right)$ denotes the bordism class of a smooth hypersurface $W_{p^{k}}$ of degree $p$ in $\mathbb{C} P^{p^{k}}$. Let $J_{n} \subseteq M U_{*}$ be the ideal $\left(w_{0}, \ldots, w_{n-1}\right)$, where $w_{0}=p$. The following statement is an important ingredient for our examples. It is a consequence of [9, Section 7; 5, Proposition 2.27].

Proposition 8.1 Let $p=2$. There is a product on $F=M U / w_{k}$ with $b_{F}\left(\bar{w}_{k} \otimes \bar{w}_{k}\right) \equiv$ $w_{k+1} \bmod J_{k}$ for $k \geqslant 0$.

\subsection{BP-theory}

We fix a prime number $p$. The Brown-Peterson spectrum $B P$ can be described as a regular quotient $B P=M U_{(p)} / I$ of the $p$-localization $M U_{(p)}$ of $M U$, where $I \subseteq\left(M U_{(p)}\right)_{*}$ is the ideal generated by the regular sequence $x_{i}, i \neq p^{k}-1, k>0$ (see $[4 ; 9])$. The coefficient ring is given by

$$
B P_{*} \cong \mathbb{Z}_{(p)}\left[v_{1}, v_{2}, \ldots\right], \quad\left|v_{i}\right|=2\left(p^{i}-1\right),
$$

where we choose the $v_{i}$ 's to be Hazewinkel's generators (see [9]).

Remark 8.2 Since $B P_{*}$ is $p$-local, we do not need to distinguish between $M U-$ products and $M U_{(p)}$-products on $B P$; see [4, Section VIII. 3].

It is shown in [9] that $B P$ is a commutative $M U$-ring. 
Proposition 8.3 There are infinitely many nonequivalent $M U$-products on $B P$, all of which induce the same ring structure in $\mathscr{D}_{\mathbb{S}}$. Infinitely many of the $M U$-products on $B P$ are commutative, but all of these are equivalent.

Proof The equivalence classes of $M U$-products on $B P$ are in one-to-one correspondence with the quadratic forms on $I / I^{2}[1]$. There are infinitely many such, for odd $p$ for instance the ones associated to the family of bilinear forms $\beta_{k}=v_{k} \bar{x}_{i(k)}^{\vee} \otimes \bar{x}_{i(k)}^{\vee}$, where $i(k)=\frac{1}{2}\left(p^{k}-1\right)$.

Let $\mu_{0}$ be a commutative product on $B P$ [9]. Any other product $\mu$ is of the form $\mu=\mu_{0} \circ \prod\left(1+a_{i j} Q_{i}^{\prime} \wedge Q_{j}^{\prime}\right)$, where $Q_{k}^{\prime} \in B P_{M U_{(p)}^{*}}^{*}(B P)$ is the Bockstein operation associated to $\bar{x}_{k}^{\vee} \in D\left(I / I^{2}[1]\right)$ (the notation $Q_{k}$ is reserved for a different Bockstein operation - see the next section). Since $B P^{*}(B P)$ is concentrated in even dimensions, all the $Q_{k}^{\prime}$ are in the kernel of the forgetful morphism

$$
B P_{M U_{(p)}}^{*}(B P) \longrightarrow B P^{*}(B P)
$$

induced by the monoidal functor $\mathscr{D}_{M U_{(p)}} \subseteq \mathscr{D}_{\mathbb{S}}$. As a consequence, all the $M U$-products on $B P$ are equal to $\mu_{0}$ in $\mathscr{D}_{\mathbb{S}}$.

The last assertion follows from Corollary 7.10.

\section{2 $P(n)$-theory}

We fix a prime number $p$ and endow $B P$ with a commutative $M U$-product. Recall that $J_{n} \subseteq M U_{*}$ is the ideal $\left(w_{0}, \ldots, w_{n-1}\right)$, where $w_{0}=p$. The sequence of the $w_{i}$ is regular, and the image of $J_{n}$ in $B P_{*}$ is the ideal $I_{n}=\left(v_{0}, \ldots, v_{n-1}\right)$, with $v_{0}=p$ (see [9]).

We define $P(n)$ as a quotient of $B P$ (see [9]):

$$
P(n)=B P / I_{n}=B P \wedge_{M U} M U / J_{n} .
$$

The coefficient ring satisfies $P(n)_{*} \cong \mathbb{F}_{p}\left[v_{n}, v_{n+1}, \ldots\right]$. The kernel $H_{n}$ of the composition $\left(M U_{(p)}\right)_{*} \rightarrow B P_{*} \rightarrow P(n)_{*}$ is generated by a regular sequence. Therefore, $P(n)=M U_{(p)} / H_{n}$ is a regular quotient of $M U_{(p)}$.

Since $P(n)_{*}$ is $p$-local, we do not need to distinguish between $M U$-products and $M U_{(p)}$-products on $P(n)$ (see Remark 8.2).

We endow $P(n)$ with an $M U$-product $\mu_{n}$ as follows. If $p$ is odd, $M U / J_{n}$ carries a commutative product $v$, since 2 is invertible. If $p=2$, we define a product $v$ on $M U / J_{n}$ as the smash ring product of the $v_{k}$ of Proposition 8.1 for $k=0, \ldots, n-1$. In any case, we define $\mu_{n}$ as the smash ring product of $\mu_{0}$, a commutative product on $B P$, with $v$. Observe that the natural map $\pi_{n}: B P \rightarrow P(n)$ is then multiplicative. 
Proposition 8.4 Let $p$ be a prime and $n \geqslant 1$. There are infinitely many nonequivalent $M U$-products on $P(n)$. All of them induce the same ring structure in $\mathscr{D}_{\mathbb{S}}$ if $p$ is odd. For $p=2$, they induce either $\mu_{n}$ or $\mu_{n}^{\mathrm{op}}$. Up to equivalence, there is a unique commutative $M U$-product for $p$ odd and no commutative $M U$-product for $p=2$.

Proof Let $P(n)$ be endowed with the product $\mu_{n}$ defined as above.

Consider first the case $p=2$. Since $v_{k} \equiv w_{k} \bmod I_{k+1}$, Proposition 8.1 and [5, Proposition 2.34] imply that $b_{P(n)}=v_{n} \bar{v}_{n-1}^{\vee} \otimes \bar{v}_{n-1}^{\vee}$. By [6] or Remark 5.6, we know that $\mu_{n}^{\mathrm{op}}=\mu_{n} \circ\left(1+v_{n} Q_{n-1} \wedge Q_{n-1}\right)$, where $Q_{n-1} \in P(n)_{M U_{(p)}}^{*}(P(n))$ is the Bockstein operation associated to $\bar{v}_{n-1}^{\vee} \in D\left(H_{n} / H_{n}^{2}[1]\right)$. By Proposition 7.12, there is no commutative product on $P(n)$.

For $p$ odd, any $M U$-product on $P(n)$ can be written as

$$
\mu_{n} \circ \prod_{i, j}\left(1+\alpha_{i j} Q_{i}^{\prime} \wedge Q_{j}^{\prime}\right)
$$

for dimensional reasons, where $Q_{k}^{\prime}$ is as in the proof of Proposition 8.3. Similarly, for $p=2$, any $M U$-product on $P(n)$ can be written as

$$
\mu_{n} \circ \prod_{i, j}\left(1+\alpha_{i j} Q_{i}^{\prime} \wedge Q_{j}^{\prime}\right) \circ\left(1+\gamma_{n} v_{n} Q_{n-1} \wedge Q_{n-1}\right)
$$

with $\gamma_{n} \in\{0,1\}$. The rest of the argument is exactly as in the proof of Proposition 8.3.

Remark 8.5 We may consider the two degenerated cases of the family $P(n), P(0)=$ $B P$ and $P(\infty)=$ hocolim $P(n)=H \mathbb{F}_{p}$, the Eilenberg-Mac Lane spectrum. The former was discussed above. For the latter, our results imply easily that it carries a unique $M U$-product, which is commutative for all $p$.

Proposition 8.6 Let $B P$ be endowed with a commutative $M U$-product. Then there are infinitely many nonequivalent $M U$-products on $P(n)$ such that the natural map $\pi_{n}: B P \rightarrow P(n)$ is multiplicative.

Proof Any product on $P(n)$ is of the form $\beta \mu_{n}$ with $\beta \in \operatorname{Bil}\left(H_{n} / H_{n}^{2}\right)$, where $\mu_{n}$ is defined as above. By Theorem 6.2, the map $\pi_{n}: B P \rightarrow \beta P(n)$ is multiplicative if and only if $P(n)_{*} \otimes b_{B P}=b_{B P}^{\beta P(n)}=\pi_{n}^{*}\left(b_{\beta P(n)}\right)$. Since $B P$ is commutative, $b_{B P}$ is trivial. Furthermore, $\pi_{n}: B P \rightarrow P(n)$ is multiplicative, by definition of $\mu_{n}$, and hence $b_{B P}^{P(n)}=0$. We then deduce from Lemma 6.6 that $\pi_{n}: B P \rightarrow \beta P(n)$ is multiplicative if and only if $\pi_{n}^{*}(\beta)=0$. We easily check that there are infinitely many such bilinear forms $\beta$ whose associated quadratic forms are different (see Remark 7.4). 


\subsection{A nondiagonalizable product}

We aim to construct a nondiagonalizable $M U$-ring spectrum.

Let $p=2, I=J_{2}=\left(w_{0}, w_{1}\right) \subseteq M U_{*}$, as above, and $F=M U / I$. Clearly, $F$ is a regular quotient $M U$-module, with $F_{*} \cong \mathbb{F}_{2}\left[x_{2}, x_{3}, \ldots\right]$. Let $\mu$ be the smash ring product of the products $v_{k}$ on $M U / w_{k}, k=0,1$, from Proposition 8.1. Let $\bar{\mu}=$ $\mu \circ\left(1+x_{2} Q_{0} \wedge Q_{1}\right)$, with $Q_{k}$ the Bockstein operation associated to $\bar{w}_{k}^{\vee} \in D\left(I / I^{2}[1]\right)$. We claim that the product $\bar{\mu}$ is not diagonalizable.

We deduce from [5, Proposition 2.34] and the construction of $\mu$ that the matrix of $b_{F}$ with respect to the basis $\bar{w}_{0}, \bar{w}_{1}$ of $I / I^{2}[1]$ is

$$
B=\left(\begin{array}{cc}
0 & 0 \\
0 & w_{2}
\end{array}\right) \text {. }
$$

From Corollary 5.3, we deduce that the matrix of $b_{\bar{F}}$ with respect to the same basis is given by

$$
\bar{B}=\left(\begin{array}{cc}
0 & x_{2} \\
x_{2} & w_{2}
\end{array}\right)
$$

Now assume that there exists an invertible matrix

$$
A=\left(\begin{array}{ll}
a & b \\
c & d
\end{array}\right)
$$

with coefficients in $F_{*}$ such that $A^{t} \bar{B} A=D$ is diagonal. We deduce from the equality above that

$$
(b c+a d) x_{2}+c d w_{2}=0 .
$$

Since $A$ is invertible, $\operatorname{det}(A)$ is a unit in $F_{*}$. Therefore $\operatorname{det}(A)=a d-b c=1$, and hence $(*)$ is equivalent to

$$
(1+2 b c) x_{2}+c d w_{2}=0 .
$$

Since $\left|x_{2}\right|=4$ and $\left|w_{2}\right|=6$, there are no coefficients in $F_{*}$ satisfying $(* *)$. Hence, $\bar{\mu}$ is not equivalent to a diagonal product, by Proposition 6.4.

\subsection{Morava $K$-theory $K(n)$}

The spectra $K(n)$ can be studied as $M U$-rings, similarly as we discussed the spectra $P(n)$ above. We adopt here a more classical point of view and work over the ground 
rings $\widehat{E}(n)$ instead. We recall the definition and the notation from there. Fix a prime number $p$. For $n>0$, there exists an $M U_{(p)}$-algebra $\widehat{E}(n)$ (see Rognes [7]) with

$$
\widehat{E}(n)_{*} \cong \lim _{k} \mathbb{Z}_{(p)}\left[v_{1}, \ldots, v_{n-1}\right]\left[v_{n}, v_{n}^{-1}\right] / I_{n}^{k},
$$

where $I_{n}$ is the ideal generated by the regular sequence $\left(v_{0}=p, v_{1}, \ldots, v_{n-1}\right)$. The $n$-th Morava $K$-theory $K(n)$ may be defined as the regular quotient of $\widehat{E}(n)$ by $I_{n}$ :

$$
K(n)=\widehat{E}(n) / I_{n} \cong \widehat{E}(n) / v_{0} \wedge_{\widehat{E}(n)} \cdots \wedge_{\widehat{E}(n)} \widehat{E}(n) / v_{n-1}
$$

Its coefficient ring satisfies $K(n)_{*} \cong \mathbb{F}_{p}\left[v_{n}, v_{n}^{-1}\right]$.

The following statement can be deduced from existing literature; see Nassau [6]. Our methods give an independent and quick proof. Let $Q_{i} \in K(n)_{\widehat{E}(n)}^{*}(K(n))$ be the Bockstein operation associated to $\bar{v}_{i}^{\vee} \in D\left(I_{n} / I_{n}^{2}[1]\right)$.

Proposition 8.7 For $p$ odd, there is precisely one $\widehat{E}(n)$-product on $K(n)$, which is commutative. For $p=2$, there are precisely two nonequivalent $\widehat{E}(n)$-products $\mu, \bar{\mu}$ on $K(n)$, both of which are noncommutative. They are related by

$$
\bar{\mu}=\mu^{\mathrm{op}}=\mu \circ\left(1+v_{n} Q_{n-1} \wedge Q_{n-1}\right)
$$

and satisfy $b_{K(n)}=b_{\bar{K}(n)}=v_{n} \bar{v}_{n-1}^{\vee} \otimes \bar{v}_{n-1}^{\vee}$.

Proof The $K(n)_{*}-$ module $I_{n} / I_{n}^{2}[1]$ is free with basis $\bar{v}_{0}, \ldots, \bar{v}_{n-1}$. Let first $p$ be odd. Because $\left|\bar{v}_{i} \otimes \bar{v}_{j}\right|<\left|v_{n}\right|$ for all $i, j<n, I_{n} / I_{n}^{2}[1]$ admits only the trivial bilinear form. Hence there is exactly one $\widehat{E}(n)$-ring structure on $K(n)$ by Theorem 4.1, which therefore must be commutative.

Let now $p=2$. For degree reasons again, there are exactly two bilinear forms on $I_{n} / I_{n}^{2}[1]$, the trivial one and $\beta=v_{n} \bar{v}_{n-1}^{\vee} \otimes \bar{v}_{n-1}^{\vee}$. Therefore, there are two $\hat{E}(n)-$ products $\mu$ and $\bar{\mu}$ on $K(n)$, related by the formula $\bar{\mu}=\beta \mu=\mu \circ\left(1+v_{n} Q_{n-1} \wedge Q_{n-1}\right)$.

Without loss of generality, we may suppose that $\mu$ is the diagonal product constructed in [5, Section 5.3], whose characteristic bilinear form $b_{K(n)}$ is $\beta$. Hence $\mu$ is noncommutative. As a consequence, we deduce $\bar{\mu}=\mu^{\mathrm{op}}$, and so $\bar{\mu}$ is noncommutative either. This is confirmed by Corollary 5.3, which implies that $b_{\bar{K}(n)}=b_{K(n)}-\left(\beta+\beta^{t}\right)=b_{K(n)}=\beta$.

Since $\beta$ is nonalternating, we find that $\mu$ and $\mu^{\text {op }}$ are not equivalent. 


\subsection{2-Periodic Morava $K$-theory $K_{n}$}

We now turn to 2-periodic Morava $K$-theory $K_{n}$. In this case, we have more products than for $K(n)$, and the situation is much more interesting.

We still fix a prime number $p$ and an integer $n>0$. There exists a commutative $\widehat{E}(n)$-algebra $E_{n}$ (see [7]), with coefficients

$$
\left(E_{n}\right)_{*} \cong \mathbb{W}\left(\mathbb{F}_{p^{n}}\right) \llbracket u_{1}, \ldots, u_{n-1} \rrbracket\left[u^{ \pm 1}\right],
$$

where $\mathbb{W}\left(\mathbb{F}_{p^{n}}\right)$ is the Witt ring on $\mathbb{F}_{p^{n}},\left|u_{i}\right|=0$ for $1 \leqslant i \leqslant n-1$ and $|u|=2$. The homomorphism induced on coefficient rings by the unit $\eta: \widehat{E}(n) \rightarrow E_{n}$ maps $v_{i}$ to $u_{i} u^{p^{i}-1}$ for $1 \leqslant i \leqslant n-1$ and $v_{n}$ to $u^{p^{n}-1}$.

Let $H_{n} \subseteq\left(E_{n}\right)_{*}$ be the ideal generated by the regular sequence $\left(u_{0}=p, u_{1}, \ldots, u_{n-1}\right)$. The 2-periodic Morava $K$-theory is defined as

$$
K_{n}=E_{n} / H_{n} \cong E_{n} / u_{0} \wedge_{E_{n}} \cdots \wedge_{E_{n}} E_{n} / u_{n-1} .
$$

Its coefficient ring satisfies $\left(K_{n}\right)_{*} \cong \mathbb{F}_{p^{n}}\left[u, u^{-1}\right]$.

Proposition 8.8 There are $p^{n} n^{2}$ different $E_{n}$-products on $K_{n}$, none is commutative for $p=2$; for $p$ odd, one is commutative if $n=1$ and $p^{n \frac{n(n-1)}{2}}$ are commutative for $n>1$.

Proof The degree zero bilinear forms

$$
H_{n} / H_{n}^{2}[1] \otimes_{\left(K_{n}\right)_{*}} H_{n} / H_{n}^{2}[1] \rightarrow\left(K_{n}\right)_{*}
$$

are in bijection with the ungraded bilinear forms

$$
H_{n} / H_{n}^{2} \otimes_{\mathbb{F}_{p^{n}}} H_{n} / H_{n}^{2} \rightarrow \mathbb{F}_{p^{n}}
$$

Hence there are $p^{n} \cdot \operatorname{dim}_{\mathbb{F}_{p^{n}}}\left(\operatorname{Bil}\left(H_{n} / H_{n}^{2}\right)\right)=p^{n} n^{2}$ different $E_{n}$-products on $K_{n}$.

For $p$ odd, there is a commutative $E_{n}$-product (see Proposition 7.8) on $K_{n}$, and the set of commutative products is in bijection with the group $\operatorname{Asym}\left(H_{n} / H_{n}^{2}\right)$, which consists of $p^{n} \frac{n(n-1)}{2}$ elements for $n>1$ and one element for $n=1$.

Let $p=2$. Using the same arguments as in the proof of [5, Proposition 5.1], we construct a diagonal product $\mu$ on $K_{n}$ with $b_{K_{n}}=u \bar{u}_{n-1}^{\vee} \otimes \bar{u}_{n-1}^{\vee}$. Hence, by Proposition 7.12, $K_{n}$ supports no commutative $E_{n}$-product.

Corollary 8.9 Up to equivalence, there are $p^{n} \frac{n(n+1)}{2}$ different $E_{n}$-products on $K_{n}$. For $p$ odd, there is a unique commutative product on $K_{n}$ up to equivalence.

Proof This is straightforward from Remark 7.4 and Proposition 7.8. 
Proposition 8.10 Any $E_{n}$-product on $K_{n}$ is diagonalizable.

Proof Apply Proposition 7.13.

\section{References}

[1] V Angeltveit, Topological Hochschild homology and cohomology of $A_{\infty}$ ring spectra, Geom. Topol. 12 (2008) 987-1032 MR2403804

[2] J M Boardman, Stable operations in generalized cohomology, from: "Handbook of algebraic topology", (I M James, editor), North-Holland, Amsterdam (1995) 585-686 MR1361899

[3] N Bourbaki, Éléments de mathématique. Première partie: Les structures fondamentales de l'analyse. Livre II: Algèbre. Chapitre 9: Formes sesquilinéaires et formes quadratiques, Actualités Sci. Ind. 1272, Hermann, Paris (1959) MR0107661

[4] A D Elmendorf, I Kriz, MA Mandell, J P May, Rings, modules, and algebras in stable homotopy theory, Math. Surveys and Monogr. 47, Amer. Math. Soc. (1997) MR1417719 With an appendix by M Cole

[5] A Jeanneret, S Wüthrich, Clifford algebras from quotient ring spectra, Manuscripta Math. 136 (2011) 33-63 MR2820395

[6] C Nassau, On the structure of $P(n)_{*} P((n))$ for $p=2$, Trans. Amer. Math. Soc. 354 (2002) 1749-1757 MR1881014

[7] J Rognes, Galois extensions of structured ring spectra. Stably dualizable groups, Mem. Amer. Math. Soc. 192, no. 898, Amer. Math. Soc. (2008) MR2387923

[8] J-P Serre, Local algebra, Springer Monogr. in Math., Springer, Berlin (2000) MR1771925 Translated from the French by C Chin and revised by the author

[9] N P Strickland, Products on MU-modules, Trans. Amer. Math. Soc. 351 (1999) 25692606 MR1641115

[10] S Wüthrich, I-adic towers in topology, Algebr. Geom. Topol. 5 (2005) 1589-1635 MR2186112

Mathematisches Institut

Sidlerstrasse 5, CH-3012 Berne, Switzerland

$S B B$

Brückfeldstrasse 16, CH-3000 Bern, Switzerland

alain.jeanneret@math.unibe.ch, samuel. wuethrich@sbb.ch

Received: 9 March 2011 